\title{
On the orbital stability of the ground states and the singularity formation for the gravitational Vlasov Poisson system
}

\author{
Mohammed Lemou*,****, Florian Méhats** and Pierre Raphael***,**** \\ * Université Paul Sabatier, MIP, Toulouse, France \\ ** IRMAR, Université Rennes 1, France \\ *** Université Paris-Sud, Orsay, France \\ **** $C N R S$
}

\begin{abstract}
We study the gravitational Vlasov Poisson system $f_{t}+v \cdot \nabla_{x} f-E \cdot \nabla_{v} f=0$ where $E(x)=\nabla_{x} \phi(x), \Delta_{x} \phi=\rho(x), \rho(x)=\int_{\mathbb{R}^{N}} f(x, v) d x d v$, in dimension $N=3,4$. In dimension $N=3$ where the problem is subcritical, we prove using concentration compactness techniques that every minimizing sequence to a large class of minimization problems attained on steady states solutions are up to a translation shift relatively compact in the energy space. This implies in particular the orbital stability in the energy space of the spherically symmetric polytropes what improves the nonlinear stability results obtained for this class in $[16,19,11]$. In dimension $N=4$ where the problem is $L^{1}$ critical, we obtain the polytropic steady states as best constant minimizers of a suitable Sobolev type inequality relating the kinetic and the potential energy. We then derive using an explicit pseudo-conformal symmetry the existence of critical mass finite time blow up solutions, and prove more generally a mass concentration phenomenon for finite time blow up solutions. This is the first result of description of a singularity formation in a Vlasov setting. The global structure of the problem is reminiscent to the one for the focusing non linear Schrödinger equation $i u_{t}=-\Delta u-|u|^{p-1} u$ in the energy space $H^{1}\left(\mathbb{R}^{N}\right)$.
\end{abstract}




\section{Introduction}

\subsection{Setting of the problem and Hamiltonian structure}

We study in this paper the gravitational Vlasov Poisson system in dimension $N=3$ or 4

$$
(V P)\left\{\begin{array}{l}
f_{t}+v \cdot \nabla_{x} f-E \cdot \nabla_{v} f=0, \quad(t, x, v) \in \mathbb{R}_{+} \times \mathbb{R}^{N} \times \mathbb{R}^{N} \\
f(t=0, x, v)=f_{0}(x, v) \geq 0, \\
\Delta_{x} \phi=\rho, \quad \phi(t, x) \rightarrow 0 \text { as }|x| \rightarrow+\infty, \\
E=\nabla_{x} \phi, \quad \rho(t, x)=\int_{\mathbb{R}^{N}} f(t, x, v) d v
\end{array}\right.
$$

In dimension $N=3$, this model describes the mechanical state of a stellar system subject to its own gravity (see for instance $[3,12]$ ). In dimension 4 , this model is also studied in the physics literature (see e.g. [8]).

Notations. For $p \in[1, \infty]$, we denote by $|\cdot|_{L^{p}}$ the $L^{p}\left(\mathbb{R}^{N}\right)$ norm as well as the $L^{p}\left(\mathbb{R}^{2 N}\right)$ norm. For any nonnegative distribution function $f(x, v), \rho_{f}$ denotes the corresponding density, $\phi_{f}$ is the Poisson potential and $E_{f}$ is the corresponding force field, these quantities being defined by

$\rho_{f}(x)=\int_{\mathbb{R}^{N}} f(x, v) d v, \quad \phi_{f}(x)=-\frac{1}{N(N-2) \omega_{N}} \int_{\mathbb{R}^{N}} \frac{1}{|x-y|^{N-2}} \rho_{f}(y) d y, \quad E_{f}=\nabla_{x} \phi_{f}$

where $\omega_{N}$ is the volume of the unit $N$-ball $\left(\omega_{3}=\frac{4 \pi}{3}\right.$ and $\left.\omega_{4}=\frac{\pi^{2}}{2}\right)$. Let

$$
p_{\text {crit }}=\frac{6 N-N^{2}}{4 N+4-N^{2}}=\left\{\begin{array}{l}
9 / 7 \text { for } N=3 \\
2 \text { for } N=4
\end{array}\right.
$$

we define for $p \in\left[p_{\text {crit }},+\infty\right]$ the energy space

$$
\mathcal{E}_{p}=\left\{f \geq 0 \text { with }|f|_{\mathcal{E}_{p}}=|f|_{L^{1}}+|f|_{L^{p}}+\left.\left.|| v\right|^{2} f\right|_{L^{1}}<+\infty\right\} .
$$

The existence of weak solutions for (1.1) in the energy space $\mathcal{E}_{p}$ is due to Horst and Hunze $[20]$ and Diperna and Lions $[9,10]$ in the more general framework of renormalized solutions. Note that, despite a number of mathematical works (see [5] and ref. therein), uniqueness is still an open problem in this framework.

Theorem 1.1 Let $N=3,4$ and $p_{\text {crit }}<p \leq+\infty$. For all $M>0$, there exists $T(M)>0$ such that for all initial data $f_{0} \in \mathcal{E}_{p}$ with $\left|f_{0}\right|_{\mathcal{E}_{p}}<M$, there exists a renormalized solution $f \in L^{\infty}\left((0, T(M)), \mathcal{E}_{p}\right)$ of (1.1) with initial data $f_{0}$. 
By a solution of (1.1), we will always mean one constructed from a standard regularization process in the framework of Theorem 1.1. Note that, in dimension $N=4$, this solution satisfies (1.1) in the distributional sense (i.e. is also a weak solution), while in dimension $N=3$, it satisfies (1.1) only for $p \geq p_{0}=(12+3 \sqrt{5}) / 11$ (see [20,9]; otherwise, if $9 / 7<p<p_{0}$, the product $E f$ may not be defined and in this case $f$ is only a renormalized solution).

These solutions verify an upper bound on the Hamiltonian

$$
\mathcal{H}(f(t))=\int_{\mathbb{R}^{2 N}}|v|^{2} f(t, x, v) d x d v-\int_{\mathbb{R}^{N}}|E(t, x)|^{2} d x \leq \mathcal{H}\left(f_{0}\right)
$$

and the conservation of the $L^{q}$ norm

$$
\forall 1 \leq q \leq p, \quad|f(t)|_{L^{q}}=\left|f_{0}\right|_{L^{q}} .
$$

From Lions and Perthame [26], some additional regularity on the initial data $f_{0}$ is propagated by the flow of (1.1) in which case (1.3) classically becomes an equality. Note that $\mathcal{E}_{p}$ is an energy space as we have the standard interpolation estimate

$$
\forall p \in\left[p_{\text {crit }},+\infty\right], \quad\left|E_{f}\right|_{L^{2}}^{2} \leq\left.\left. C_{p}|| v\right|^{2} f\right|_{L^{1}} ^{\frac{N-2}{2}}|f|_{L^{1}}^{\frac{4 N+4-N^{2}}{2 N(p-1)}\left(p-p_{\text {crit }}\right)}|f|_{L^{p}}^{\frac{p(N-2)}{N(p-1)}} .
$$

(1.1) also admits a number of symmetries in the energy space $\mathcal{E}_{p}$ :

- Space-time translation invariance: if $f(t, x, v)$ solves $(1.1)$, then $\forall\left(t_{0}, x_{0}\right) \in \mathbb{R} \times \mathbb{R}^{N}$, so does $f\left(t+t_{0}, x+x_{0}, v\right)$.

- Scaling invariance: if $f(t, x, v)$ solves $(1.1)$, then $\forall\left(\lambda_{0}, \mu_{0}\right) \in \mathbb{R}_{*}^{+} \times \mathbb{R}_{+}^{*}$, so does

$$
\frac{\mu_{0}^{N-2}}{\lambda_{0}^{2}} f\left(\frac{t}{\lambda_{0} \mu_{0}}, \frac{x}{\lambda_{0}}, \mu_{0} v\right)
$$

- Galilean invariance: if $f(t, x, v)$ solves (1.1), then $\forall v_{0} \in \mathbb{R}^{N}$, so does $f\left(t, x+v_{0} t, v+\right.$ $\left.v_{0}\right)$.

In dimension $N=3$, solutions built from Theorem 1.1 are global $(T(M)=+\infty$ for any $M$ ) and bounded in $\mathcal{E}_{p}$ as the bound on the Hamiltonian and the $L^{p}$ norm together with (1.5) and $\frac{N-2}{2}<1$ imply a uniform upper bound on the kinetic energy.

In dimension $N=4$, the global existence of solutions is ensured for small initial data (see, e.g., $[9,10])$. For arbitrary large data, the Cauchy problem is well posed locally in time with a lifespan lower bounded by a function of the size of the initial data only. For the sake of completeness, a proof of this last fact is sketched in Appendix C. Additionnaly, it is well known from the virial identity that blow up can occur, see [15]. Indeed, let $f_{0}$ compactly supported and $f(t)$ the corresponding strong solution to $(1.1)$ on $[0, T)$, then

$$
\forall t \in[0, T), \quad \frac{d^{2}}{d t^{2}} \int_{\mathbb{R}^{2 N}}|x|^{2} f(x, v) d x d v=\mathcal{H}\left(f_{0}\right) .
$$


Thus if $\mathcal{H}\left(f_{0}\right)<0$, then the positive quantity $\int_{\mathbb{R}^{2 N}}|x|^{2} f(x, v) d x d v$ must become negative in finite time and $f$ blows up in finite time. Moreover, we say that (1.1) is critical in the sense that the strength of the kinetic and the potential energy exactly balance from (1.5).

\subsection{A parallel with the nonlinear Schrödinger equation}

The Hamiltonian and symmetries structure of (1.1) is reminiscent to the one for the focusing nonlinear Schrödinger equation

$$
(N L S)\left\{\begin{array}{l}
i u_{t}=-\Delta u-|u|^{p-1} u, \quad(t, x) \in[0, T) \times \mathbb{R}^{N} \\
u(0, x)=u_{0}(x), \quad u_{0}: \mathbb{R}^{N} \rightarrow \mathbb{C}
\end{array}\right.
$$

with $u_{0} \in H^{1}=H^{1}\left(\mathbb{R}^{N}\right)$ in dimension $N \geq 1$. For $1<p<+\infty$ if $N=1,2$, or $1<p<\frac{N+2}{N-2}$ if $N \geq 3,(1.8)$ is locally well-posed in the energy space $H^{1}$ from [14]. These solutions verify the conservation of the Hamiltonian

$$
\mathcal{H}(u(t))=\frac{1}{2} \int|\nabla u(t, x)|^{2} d x-\frac{1}{p+1} \int|u(t, x)|^{p+1} d x=\mathcal{H}\left(u_{0}\right)
$$

and the $L^{2}$ mass

$$
|u(t)|_{L^{2}}=\left|u_{0}\right|_{L^{2}}
$$

and (1.8) is invariant through the same group of $H^{1}$ symmetries: if $u(t, x)$ solves $(1.8)$, then $\forall\left(\lambda_{0}, t_{0}, x_{0}, \beta_{0}, \gamma_{0}\right) \in \mathbb{R}_{*}^{+} \times \mathbb{R} \times \mathbb{R}^{N} \times \mathbb{R}^{N} \times \mathbb{R}$, so does $v(t, x)=\lambda_{0}^{\frac{2}{p-1}} u\left(t+t_{0}, \lambda_{0} x+\right.$ $\left.x_{0}-\beta_{0} t\right) e^{i \frac{\beta_{0}}{2} \cdot\left(x-\frac{\beta_{0}}{2} t\right)} e^{i \gamma_{0}}$. The space $H^{1}$ is the energy space from the Gagliardo-Nirenberg inequality

$$
\int|u(t, x)|^{p+1} d x \leq C_{p}|\nabla u|_{L^{2}}^{\frac{N(p-1)}{2}}|u|_{L^{2}}^{p+1-\frac{N}{2}(p-1)} .
$$

Thus, if $\frac{N(p-1)}{2}<2$ i.e. $p<1+\frac{4}{N}$, all solutions to (1.8) are global and bounded in $H^{1}$. On the contrary, in the critical case $p=1+\frac{4}{N}$, the virial identity

$$
\frac{d^{2}}{d t^{2}} \int|x|^{2}|u(t, x)|^{2}=16 \mathcal{H}\left(u_{0}\right)
$$

implies finite time blow up for $\mathcal{H}\left(u_{0}\right)<0$, see [39], and the problem is $L^{2}$ critical as the $L^{2}$ norm is conserved and all symmetries are $L^{2}$ isometries.

Special solutions play a fundamental role in the description of the dynamics of (1.8) both in the subcritical case $p<1+\frac{4}{N}$ and the critical case $p=1+\frac{4}{N}$. They are the so-called solitary waves of the form $u(t, x)=e^{i \omega t} W_{\omega}(x), \omega>0$, where $W_{\omega}$ solves

$$
\Delta W_{\omega}+W_{\omega}\left|W_{\omega}\right|^{p-1}=\omega W_{\omega}
$$


Equation (1.10) is a standard nonlinear elliptic equation and from [2], [13] and [21], there is a unique positive solution up to translation $G_{\omega}^{p, N}$ which is in addition radially symmetric. Letting $G^{p, N}=G_{1}^{p, N}$, then $G_{\omega}^{p, N}(x)=\omega^{\frac{p-1}{4}} G^{p, N}\left(\omega^{\frac{1}{2}} x\right)$ from scaling property. Moreover, Weinstein in [38] proved the following variational characterization of $G^{p, N}$ : the minimization problem

$$
\tilde{J}_{p, N}=\inf _{u \in H^{1}, u \neq 0} \frac{|u|_{L^{2}}^{p+1-\frac{N}{2}(p-1)}|\nabla u|_{L^{2}}^{\frac{N(p-1)}{2}}}{|u|_{L^{p+1}}^{p+1}}
$$

is attained exactly on the four parameters family

$$
a_{0} G^{p, N}\left(\lambda_{0} x+x_{0}\right) e^{i \gamma_{0}}, \quad\left(a_{0}, \lambda_{0}, x_{0}, \gamma_{0}\right) \in \mathbb{R} \times \mathbb{R}_{*}^{+} \times \mathbb{R}^{N} \times \mathbb{R} .
$$

Note from (1.9) that (1.11) amounts comparing the strength of the kinetic and the potential energy in the Hamiltonian.

A more refined result has been obtained by Cazenave and Lions, [7], using the concentration compactess techniques introduced by P.-L. Lions in [24], [25].

Theorem $1.2([7])$ Let $p<1+\frac{4}{N}$. For any $M>0$, the minimization problem

$$
\inf _{u \in H^{1},|u|_{L^{2}}=M} \mathcal{H}(u)
$$

is attained exactly on the two parameters family

$$
\lambda_{p, N}^{\frac{2}{p-1}} G^{p, N}\left(\lambda_{p, N} x+x_{0}\right) e^{i \gamma_{0}}, \quad\left(x_{0}, \gamma_{0}\right) \in \mathbb{R}^{N} \times \mathbb{R}, \quad \lambda_{p, N}=\left(\frac{M}{\left|G^{p, N}\right|_{L^{2}}}\right)^{\frac{2(p-1)}{4 N-N(p-1)}} .
$$

Moreover, any minimizing sequence for (1.12) is relatively compact in $H^{1}$ up to a translation and a phase shift.

A fundamental corollary is the so-called orbital stability of the ground state solitary wave for $p<1+\frac{4}{N}$ : $\forall \varepsilon>0, \exists \delta(\varepsilon)>0$ such that $\forall u_{0} \in H^{1}$ with

$$
\left|\mathcal{H}\left(u_{0}\right)-\mathcal{H}\left(G^{p, N}\right)\right|+\left.|| u_{0}\right|_{L^{2}}-\left|G^{p, N}\right|_{L^{2}} \mid<\delta(\varepsilon),
$$

there exists $x(t) \in \mathbb{R}^{N}, \gamma(t) \in \mathbb{R}$ such that the corresponding solution $u(t)$ to (1.8) satisfies

$$
\forall t \in[0,+\infty), \quad\left|u(t, \cdot+x(t)) e^{i \gamma(t)}-G^{p, N}\right|_{H^{1}}<\varepsilon .
$$

This is indeed a straightforward consequence of Theorem 1.2 and the conservation of the Hamiltonian and the $L^{2}$ norm. Let us say that the orbital stability in the energy space is a fundamental feature which is the starting point of a more refined analysis of the large time 
behavior of the solution in the presence of solitons, see for example the works by Martel and Merle for the subcritical KdV equation [27], and also Martel, Merle and Tsai, [28].

Theorem 1.2 is certainly false for $p=1+\frac{4}{N}$ as due to the $L^{2}$ scaling invariance, all ground states have the same $L^{2}$ mass. Nevertheless, the recent works by Merle and Raphael, see for example [30], [31], show that it is at the heart of the description of the singularity formation. For example, these authors exhibit a class of initial data in $H^{1}$ which blow up in finite time $0<T<+\infty$ with a blow up profile given exactly by the ground state $G^{1+\frac{4}{N}, N}$. Note that some nonlinear stability result on the ground state in the energy space is here again the starting point of the analysis.

\subsection{Statements of the results}

We look for stationary solutions to (1.1) and observe that if $f(t, x, v)=F(e)$ where the microscopic energy is

$$
e=\frac{|v|^{2}}{2}+\phi(x)
$$

then $f$ is a solution to the stationary (VP) system. In dimension $N=3$, a well known class of steady states of physical relevance are the so-called polytropes

$$
f(x, v)=\left(e_{0}-e\right)_{+}^{k}
$$

for some energy threshold $e_{0}$ and $0<k \leq \frac{7}{2}$, and where we note $g_{+}=\max (g, 0)$. We refer to [19] and references therein for a complete introduction to the history of the problem. In dimension $N=3$, a large class of radial steady states has been constructed solving the associated non linear radial ODE in [1]. More recently in [16, 17, 18, 35], part of these steady states including the polytropes have been obtained as minimizers of appropriately chosen energy-Casimir functionals under a constraint of prescribed mass. In particular, Guo and Rein proved the following in [19]: given $M>0$, the minimization problem

$$
\inf _{f \geq 0, \quad \int_{\mathbb{R}^{6}} f+\int_{\mathbb{R}^{6}} j(f)=M} \mathcal{H}(f)
$$

is attained on a steady state solution to (1.1); moreover, every minimizing sequence is up to a translation shift weakly relatively compact in some $L^{p}, p>1$ - see Theorem 1 in [19]from which a dynamical nonlinear stability statement is derived and eventually completed by Schaeffer in [37]. Let us insist onto the following points:

(i) First, strategy (1.14) would fail to build steady states for $N=4$ due to the $L^{1}$ scaling invariance which leaves any term like $\int_{\mathbb{R}^{2 N}} j(f)$ invariant. In the special case $j(f)=f^{k}$, there are in fact simpler ways of deriving a minimization problem solved by the polytropic steady state than (1.14) which suffers from the lack of compactness due to translation invariance. In particular, an adaptation of the approaches in [2] or [38] will be succesfull for $N=3$ and $N=4$. 
(ii) The nonlinear stability statement of the obtained steady states in $[16,19,11,37]$ is measured in terms of a distance which under some very specific constraints on the perturbation is proved to control the $L^{2}$ norm. One of the difficulties the authors are confronted with is the weak $L^{p}$ convergence of the minimizing sequences of (1.14) which is a consequence of their strategy ie their choice of minimization problem.

Our aim in this paper is to view the problem through the standard concentration compactness techniques introduced by P.-L. Lions in [24], [25], and to derive the natural orbital stability statements in this frame both in dimensions $N=3$ and $N=4$. An additional striking feature in dimension $N=4$ will be the existence of an explicit pseudoconformal symmetry (similar to the one given in [4] for the Vlasov-Manev system in dimension 3) which allows one to exhibit explicit critical mass finite time blow up solutions like for the $L^{2}$ critical nonlinear Schrödinger equation. The results stated in this text were announced in the Note [22].

\subsubsection{Variational characterization of the polytropes}

We first claim that in the homogeneous case $j(f)=f^{k}$, the spherically symmetric polytropes may be attained in dimension $N=3,4$ as minimizers of a suitable Sobolev type inequality as in [38]. To wit, let us fix some notations. For $p \in\left(p_{\text {crit }},+\infty\right]$, we let $\phi_{p}$ be the unique radial solution to

$$
-\frac{1}{r^{N-1}} \frac{d}{d r}\left(r^{N-1} \phi_{p}^{\prime}\right)+\gamma_{N, p}\left(-1-\phi_{p}\right)_{+}^{\frac{1}{p-1}+\frac{N}{2}}=0, \quad \phi_{p}(r) \rightarrow 0 \quad \text { as } \quad r \rightarrow+\infty,
$$

with

$$
\gamma_{N, p}=N \omega_{N} \int_{0}^{1}(2 t)^{\frac{N-2}{2}}(1-t)^{\frac{1}{p-1}} d t .
$$

Note that the uniqueness of this object follows from the scaling invariance of (1.15). The corresponding spherically symmetric polytropic steady state is

$$
Q_{p}(x, v)=\left\{\begin{array}{l}
(-1-e)^{\frac{1}{p-1}} \text { for } e<-1 \\
0 \text { for } e>-1
\end{array}\right.
$$

We characterize $Q_{p}$ in terms of a best constant in interpolation estimate (1.5).

Theorem 1.3 (Variational characterization of $Q_{p}$ ) Let $N=3,4$ and $p_{\text {crit }}<p \leq$ $+\infty$. The minimization problem

$$
J_{N, p}=\inf _{f \in \mathcal{E}_{p}, \quad f \neq 0} \frac{\left.\left.|| v\right|^{2} f\right|_{L^{1}} ^{\frac{N-2}{2}}|f|_{L^{1}}^{\frac{4 N+4-N^{2}}{2 N(p-1)}\left(p-p_{c r i t}\right)}|f|_{L^{p}}^{\frac{p(N-2)}{N(p-1)}}}{|E|_{L^{2}}^{2}}
$$

is attained exactly on the four parameters family

$$
\gamma Q_{p}\left(\frac{x-x_{0}}{\lambda}, \mu v\right), \quad\left(\gamma, \lambda, \mu, x_{0}\right) \in \mathbb{R}_{+}^{*} \times \mathbb{R}_{+}^{*} \times \mathbb{R}_{+}^{*} \times \mathbb{R}^{N} .
$$




\subsubsection{Orbital stability of the polytropes in dimension $N=3$}

We now turn in dimension $N=3$ to the question of the dynamical stability of $Q_{p}$. First observe from a straightforward rescaling argument that Theorem 1.3 for $N=3$ is equivalent to the following: given $\frac{9}{7}<p \leq+\infty$ and $M_{1}, M_{p}>0$, the minimization problem

$$
\inf _{f \in \mathcal{E}_{p}, \quad|f|_{L^{1}}=M_{1}, \quad|f|_{L^{p}}=M_{p}} \mathcal{H}(f)
$$

is attained on the one parameter family

$$
\frac{\mu}{\lambda^{2}} Q_{p}\left(\frac{x-x_{0}}{\lambda}, \mu v\right), \quad x_{0} \in \mathbb{R}^{N}
$$

with

$$
\lambda=\left(\frac{M_{p}}{\left|Q_{p}\right|_{L^{p}}}\right)^{\frac{2 p}{9(p-1)}}\left(\frac{\left|Q_{p}\right|_{L^{1}}}{M_{1}}\right)^{\frac{7 p-9}{9(p-1)}}, \frac{\lambda}{\mu}=\left(\frac{M_{p}}{\left|Q_{p}\right|_{L^{p}}}\right)^{\frac{p}{3(p-1)}}\left(\frac{M_{1}}{\left|Q_{p}\right|_{L^{1}}}\right)^{\frac{p}{3(p-1)}} .
$$

From the control of the energy (1.3) and the $L^{q}$ norm (1.4), the question of the non linear stability of $Q_{p}$ through the flow of (1.1) may be answered by proving compactness results for the minimizing sequences of (1.19). Using standard concentration compactness techniques, one can prove in fact in a much more general frame the compactness in the energy space of the minimizing sequences.

Theorem 1.4 (Compactness of the minimizing sequences) Let $N=3$.

(i) Let $j$ a strictly convex continuous nonnegative function on $\mathbb{R}_{+}$with

$$
\forall t \geq 0, \quad j(t) \geq C t^{p} \quad \text { with } \quad p_{\text {crit }}<p<+\infty \quad \text { and } \lim _{t \rightarrow 0} \frac{j(t)}{t}=0 .
$$

Given $M_{1}, M_{j}>0$, let

$$
\mathcal{F}\left(M_{1}, M_{j}\right)=\left\{f \in \mathcal{E}_{p} \quad \text { with } \int_{\mathbb{R}^{6}} f=M_{1}, \quad \int_{\mathbb{R}^{6}} j(f)=M_{j}\right\}
$$

and

$$
I\left(M_{1}, M_{j}\right)=\inf _{f \in \mathcal{F}\left(M_{1}, M_{j}\right)} \mathcal{H}(f) .
$$

Assume that the non dichotomy condition is fulfilled: $\forall 0<\alpha<1, \forall 0 \leq \beta \leq 1$,

$$
I\left(M_{1}, M_{j}\right)<I\left(\alpha M_{1}, \beta M_{j}\right)+I\left((1-\alpha) M_{1},(1-\beta) M_{j}\right),
$$

then for every minimizing sequence $\left(f_{n}\right)_{n \geq 1}$ of (1.23), there exists $\left(y_{n}\right)_{n \geq 1}$ in $\mathbb{R}^{3}$ such that $f_{n}\left(x+y_{n}, v\right)$ is relatively compact in the energy space $\mathcal{E}_{p}$ and converges to a minimizer.

(ii) Similarly, let $f_{n} \in \mathcal{E}_{\infty}$ be a minimizing sequence for

$$
I\left(M_{1}, M_{\infty}\right)=\inf _{|f|_{L^{1}}=M_{1}, \quad|f|_{L^{\infty}=M_{\infty}}} \mathcal{H}(f),
$$

then there exists $\left(y_{n}\right)_{n \geq 1}$ in $\mathbb{R}^{3}$ such that up to a subsequence, $f_{n}\left(x+y_{n}, v\right) \rightarrow f$ in $L^{1}$ and $L^{\infty}$ weak $\star$ where $f$ is given by (1.20), (1.21) for $p=\infty$. 
Non dichotomy condition (1.24) is the standard type of control at the heart of the concentration compactness techniques, see [24]. We do not know whether it is fulfilled or not in general. We nevertheless can obtain from scaling arguments the following sufficient criteria.

Lemma 1.5 (Sufficient conditions to ensure (1.24)) Assume one of the following: (i) $j$ is a polytrope ie

$$
j(f)=f^{p} \text { with } \frac{9}{7}<p<+\infty ;
$$

(ii) there exists $\frac{3}{2}<p_{1} \leq p_{2}<+\infty$ such that

$$
\forall t \geq 0, \quad \forall b \geq 1, \quad b^{p_{1}} j(t) \leq j(b t) \leq b^{p_{2}} j(t) ;
$$

then (1.24) holds.

From the Hamiltonian structure of (1.1), Theorem 1.4 classically implies the orbital stability in the energy space of the family of minimizers of (1.23) through the flow of (1.1). Minimizers are radial up to a translation shift because symmetric rearrangements increase the potential energy, see Appendix B, and one can prove following [19] that they are steady states solutions to (1.1); but uniqueness of the radial minimizer of (1.23) is not known in general. It is nevertheless straightforward in the homogeneous case $j(f)=f^{p}$ due to the scaling invariance of the obtained Euler-Lagrange equations in this case. We thus have the orbital stability of $Q_{p}$ in the energy space $\mathcal{E}_{p}$ for $p_{\text {crit }}<p<+\infty$.

Corollary 1.6 (Orbital stability of $Q_{p}$ in $\mathcal{E}_{p}$ ) Let $N=3$ and $p_{\text {crit }}<p<+\infty$. Then for all $\varepsilon>0$, there exists $\delta(\varepsilon)>0$ such that the following holds true. Let $f_{0} \in \mathcal{E}_{p}$ with

$$
\begin{gathered}
\mathcal{H}\left(f_{0}\right)-\mathcal{H}\left(Q_{p}\right) \leq \delta(\varepsilon), \\
\left|f_{0}\right|_{L^{1}} \leq\left|Q_{p}\right|_{L^{1}}+\delta(\varepsilon), \quad\left|f_{0}\right|_{L^{p}} \leq\left|Q_{p}\right|_{L^{p}}+\delta(\varepsilon),
\end{gathered}
$$

and let $f(t) \in L^{\infty}\left(\mathbb{R}_{+}, \mathcal{E}_{p}\right)$ a weak solution to (1.1) with initial data $f_{0}$, then there exists a translation shift $x(t) \in \mathbb{R}^{N}$ such that $\forall t \in[0,+\infty)$,

$$
\left|f(t, x+x(t), v)-Q_{p}\right|_{\mathcal{E}_{p}}<\varepsilon .
$$

This result is a direct consequence of the strong convergence of minimizing sequences, stated in Proposition 4.3. In the case $p=+\infty$, this strategy only provides a weak convergence result (see also Proposition 4.3), which cannot be translated into (strong) orbital stability for the ground state $Q_{\infty}$.

Remark 1.7 Very recently and independently from this work, Sánchez and Soler, [36], have obtained the orbital stability of $Q_{p}$ in dimension $N=3$ in $L^{1} \cap L^{p}, \frac{9}{7}<p<+\infty$. Note also that we systematically leave aside the case $p=p_{\text {crit }}=\frac{9}{7}$ where additional invariances occur and which would require a slightly different analysis. We refer to [19] for some stability results in this case. 


\subsubsection{Singularity formation and mass concentration for $N=4$}

In dimension $N=4$, following [38], we first give a criterion for the global wellposedness of the Cauchy problem.

Corollary 1.8 (Global wellposedness criterion) Let $N=4$ and $p_{\text {crit }}<p \leq+\infty$. Let $f_{0} \in \mathcal{E}_{p}$ with

$$
\left|f_{0}\right|_{L^{1}}\left|f_{0}\right|_{L^{p}}^{\frac{p}{p-2}}<\left|Q_{p}\right|_{L^{1}}\left|Q_{p}\right|_{L^{p}}^{\frac{p}{p-2}}
$$

then there exists a global and bounded weak solution to (1.1) with initial data $f_{0}$ in $\mathcal{E}_{p}$.

Remark 1.9 Let us observe that the quantity $|f|_{L^{1}}|f|_{L^{p}}^{\frac{p}{p-2}}$ is invariant through the scaling transformation (1.6).

The proof of Corollary 1.8 is straightforward from Theorem 1.3. Indeed, first observe that $Q_{p}$ is a well localized steady solution to (1.1), and thus (1.7) implies $\mathcal{H}\left(Q_{p}\right)=0$. We conclude from (1.18): $\forall f \in \mathcal{E}_{p}$,

$$
\mathcal{H}(f) \geq\left.\left.|| v\right|^{2} f\right|_{L^{1}}\left(1-\left(\frac{|f|_{L^{1}}|f|_{L^{p}}^{\frac{p}{p-2}}}{\left|Q_{p}\right|_{L^{1}}\left|Q_{p^{p}}\right|_{L^{p}}^{\frac{p}{p-2}}}\right)^{\frac{p-2}{2(p-1)}}\right) .
$$

Local weak solutions built from Theorem 1.1 thus admit from (1.3) and (1.4) a uniform bound on the kinetic energy and may be continued to yield a global weak solution.

A striking feature is that as it is the case for the $L^{2}$ critical nonlinear Schrödinger equation, the global wellposedness criterion of Corollary 1.8 is sharp as there exist critical mass blow up solutions. This is a consequence of the pseudo-conformal symmetry. Indeed, let us observe that if $f(t, x, v)$ is a solution to (1.1), then $\forall a \in \mathbb{R}$, so is

$$
f_{a}(t, x, v)=f\left(\frac{t}{1-a t}, \frac{x}{1-a t},(1-a t) v+a x\right) .
$$

Remark 1.10 Of course, the group of symmetries of the linear Vlasov equation and the linear Schrödinger equation are the same as an explicit transformation, the Wigner transform, allows one to pass from the one to the other. In the case of the Schrödinger equation, the pseudo-conformal symmetry is

$$
u_{a}(t, x)=\frac{1}{(1-a t)^{\frac{N}{2}}} u\left(\frac{t}{1-a t}, \frac{x}{1-a t}\right) e^{\frac{i|x|^{2}}{4(1-a t)}} .
$$

Now a striking feature is that exactly like for the $L^{2}$ critical (NLS) (or for the VlasovManev system in dimension 3 [4]), the pseudo conformal symmetry is in the critical case still a symmetry of the non linear problem. 
We thus apply (1.29) to the polytrope $Q_{p}$ to obtain a critical mass blow up solution to $(1.1)$

$$
S_{p}(t, x, v)=Q_{p}\left(\frac{x}{1-t},(1-t) v+x\right)
$$

which satisfies

- $\left|S_{p}\right|_{L^{1}}=\left|Q_{p}\right|_{L^{1}},\left|S_{p}\right|_{L^{p}}=\left|Q_{p}\right|_{L^{p}}$.

- $S_{p}(t)$ blows up at time $t=1$ with speed

$$
\left.\left.|| v\right|^{2} S_{p}(t)\right|_{L^{1}} ^{1 / 2} \sim \frac{\left.\left.|| v\right|^{2} Q_{p}\right|_{L^{1}} ^{1 / 2}}{1-t}
$$

- $S_{p}(t)$ leaves $\mathcal{E}_{p}$ by leaving $L^{1}$ and forming a Dirac mass. Indeed, $\rho_{S_{p}}(x)=\int_{\mathbb{R}^{4}} S_{p}(t, x, v) d v$ satisfies

$$
\rho_{S_{p}} \rightarrow\left|Q_{p}\right|_{L^{1}} \delta_{x=0}
$$

in the weak sense of measures. Similarly, $\rho_{S_{p}, p}(x)=\left(\int_{\mathbb{R}^{4}} S_{p}^{p}(t, x, v) d v\right)^{1 / p}$ satisfies for $p<+\infty$

$$
\rho_{S_{p}, p}^{p} \rightarrow\left|Q_{p}\right|_{L^{p}}^{p} \delta_{x=0}
$$

in the weak sense of measures.

We now claim that $Q_{p}$ for $p_{\text {crit }}<p<+\infty$ is orbitally stable up to an additional rescaling of the solution. First observe from Theorem 1.3 and a straightforward rescaling argument that $Q_{p}$ admits the following variational characterization: if $f \in \mathcal{E}_{p}$ satisfies

$$
\mathcal{H}(f)=0 \text { and }|f|_{L^{1}}|f|_{L^{p}}^{\frac{p}{p-2}}=\left|Q_{p}\right|_{L^{1}}\left|Q_{p}\right|_{L^{p}}^{\frac{p}{p-2}},
$$

then there exists $\left(\lambda, \mu, x_{0}\right) \in \mathbb{R}_{*}^{+} \times \mathbb{R}_{*}^{+} \times \mathbb{R}^{4}$ such that

$$
f(x, v)=\left(\frac{\mu}{\lambda}\right)^{2} Q_{p}\left(\frac{x-x_{0}}{\lambda}, \mu v\right) .
$$

We now claim the orbital stability of $Q_{p}$, see for example [30] for the Schrödinger case.

Proposition 1.11 (Orbital stability of $Q_{p}$ in a blow up setting) Let $N=4$ and $p_{\text {crit }}<$ $p<+\infty$. Then for all $\varepsilon>0$, there exists $\delta(\varepsilon)>0$ such that the following holds true. Let $f_{0} \in \mathcal{E}_{p}$ with

$$
\left|f_{0}\right|_{L^{1}} \leq\left|Q_{p}\right|_{L^{1}}+\delta(\varepsilon), \quad\left|f_{0}\right|_{L^{p}} \leq\left|Q_{p}\right|_{L^{p}}+\delta(\varepsilon)
$$

and $f(t) \in \mathcal{E}_{p}, t \in[0, T)$, a weak solution to (1.1) with initial data $f_{0}$ such that

$$
\forall t \in[0, T), \quad \lambda^{2}(t) \mathcal{H}(f(t))<\delta(\varepsilon)
$$


where

$$
\lambda(t)=\left(\frac{\left.\left.|| v\right|^{2} Q_{p}\right|_{L^{1}}}{\left.\left.|| v\right|^{2} f(t)\right|_{L^{1}}}\right)^{\frac{1}{2}} .
$$

Then there exists a translation shift $x(t) \in \mathbb{R}^{N}$ such that $\forall t \in[0, T)$,

$$
\left|f\left(t, \lambda(t)(x+x(t)), \frac{v}{\lambda(t)}\right)-Q_{p}\right|_{\mathcal{E}_{p}}<\varepsilon .
$$

Note that from Theorem 1.1, finite time blow up is equivalent to

$$
\left.\left.\lim _{t \rightarrow T}|| v\right|^{2} f(t)\right|_{L^{1}}=+\infty
$$

and then the upper bound on the Hamiltonian (1.3) implies that (1.30) holds close to blow up time. Another simple way to fulfill $(1.30)$ is to assume $\mathcal{H}\left(f_{0}\right)<0$. This results thus asserts that the blow up profile of small super critical mass blow up solutions must be close to the ground state in the $\mathcal{E}_{p}$ sense. Again, this fundamental rigidity property is the starting point of the blow up analysis for (NLS) in [30].

Following Merle and Tsutsumi, [33], and Nawa, [34], we eventually claim from the variational characterization of $Q_{p}$ a mass concentration phenomenon for finite time blow up solutions to (1.1).

Theorem 1.12 (Mass concentration in $L^{1}$ and $L^{p}$ ) Let $N=4$ and $p_{\text {crit }}<p<+\infty$. Let $f_{0} \in \mathcal{E}_{p}$ and $f(t) \in \mathcal{E}_{p}, t \in[0, T)$, a weak solution to (1.1) with initial data $f_{0}$. Assume that $f(t)$ blows up in finite time $0<T<+\infty$ ie

$$
\left.\left.|| v\right|^{2} f(t)\right|_{L^{1}} \rightarrow+\infty \quad \text { as } \quad t \rightarrow T \text {. }
$$

Let $\rho(t, x)=\int_{\mathbb{R}^{4}} f(t, x, v) d v$ and $\rho_{p}(t, x)=\left(\int_{\mathbb{R}^{4}} f^{p}(t, x, v) d v\right)^{\frac{1}{p}}$. Then there exists a translation shift $x(t) \in \mathbb{R}^{N}$ such that $\forall R>0$,

$$
M_{1}(R)=\liminf _{t \rightarrow T} \int_{|x-x(t)|<R} \rho(t, x) d x, \quad M_{p}(R)=\liminf _{t \rightarrow T}\left(\int_{|x-x(t)|<R} \rho_{p}^{p}(t, x) d x\right)^{\frac{1}{p}}
$$

satisfy

$$
M_{1}(R)\left(M_{p}(R)\right)^{\frac{p}{p-2}} \geq\left|Q_{p}\right|_{L^{1}}\left|Q_{p}\right|_{L^{p}}^{\frac{p}{p-2}}
$$

Recently, Merle and Raphael, [32], proved that small super critical mass solution to the critical non linear Schrödinger equation (1.8) focus exactly the quantized and universal amount of $L^{2}$ mass $\left|G^{1+\frac{4}{N}, N}\right|_{L^{2}}$ at blow up time. This type of question now arises naturally from Theorem 1.12 and is widely open for (1.1). 


\section{Variational characterization of the polytropes for $N=3,4$}

This section is devoted to the proof of Theorem 1.3. For any $f \in \mathcal{E}_{p} \backslash\{0\}$ and for $p \in$ $\left(p_{\text {crit }}, \infty\right]$, we set

$$
J_{N, p}(f)=\frac{\left.\left.|| v\right|^{2} f\right|_{L^{1}} ^{\alpha_{1}}|f|_{L^{1}}^{\alpha_{2}}|f|_{L^{p}}^{\alpha_{3}}}{\left|E_{f}\right|_{2}^{2}}
$$

with

$$
\alpha_{1}=\frac{N-2}{2} \quad \alpha_{2}=\frac{4 N+4-N^{2}}{2 N(p-1)}\left(p-p_{\text {crit }}\right), \quad \alpha_{3}=\frac{p(N-2)}{N(p-1)} .
$$

In particular, for $N=3$ or $N=4$ we have

$$
J_{3, p}(f)=\frac{\|\left.\left. v\right|^{2} f\right|_{L^{1}} ^{1 / 2}|f|_{L^{1}}^{\frac{7 p-9}{6(p-1)}}|f|_{L^{p}}^{\frac{p}{3(p-1)}}}{\left|E_{f}\right|_{L^{2}}^{2}}, \quad J_{4, p}(f)=\frac{\|\left.\left. v\right|^{2} f\right|_{L^{1}}|f|_{L^{1}}^{\frac{p-2}{2(p-1)}}|f|_{L^{p}}^{\frac{p}{2(p-1)}}}{\left|E_{f}\right|_{L^{2}}^{2}} .
$$

The interpolation estimate (1.5) shows that the functional $J_{N, p}$ is bounded from below by a positive constant. Consider the best constant in this interpolation estimate:

$$
J_{N, p}=\inf _{f \in \mathcal{E}_{p} \backslash\{0\}} J_{N, p}(f) .
$$

Our aim in this section is to show that $J_{N, p}$ is attained and to characterize the corresponding minimizers. For the sake of clarity, the proof of Theorem 1.3 is decomposed into several steps developed in subsections 2.1, 2.2 and 2.3.

\subsection{Existence of the minimizers}

This first part of the proof is adapted from Weinstein's argument [38]. Consider a minimizing sequence $f_{n}$ for $J_{N, p}$. First remark using the formula given in Appendix A that for any $f \in \mathcal{E}_{p} \backslash\{0\}$, the rescaling

$$
f(x, v) \mapsto\left(T_{\gamma, \lambda, \mu} f\right)(x, v)=\gamma f\left(\frac{x}{\lambda}, \mu v\right), \quad(\gamma, \lambda, \mu) \in\left(\mathbb{R}_{+}^{*}\right)^{3}
$$

leaves $J_{N, p}$ invariant

$$
J_{N, p}\left(T_{\gamma, \lambda, \mu} f\right)=J_{N, p}(f) .
$$

By choosing

$$
\gamma=|f|_{L^{1}}^{-\frac{1}{p-1}}|f|_{L^{p}}^{-\frac{p}{p-1}}, \quad \lambda=\left.\left.|f|_{L^{1}}^{-\frac{(N+2) p-N}{2 N(p-1)}}|f|_{L^{p}}^{\frac{p}{N(p-1)}}|| v\right|^{2} f\right|_{L^{1}} ^{1 / 2}, \quad \mu=\left.\left.|f|_{L^{1}}^{-1 / 2}|| v\right|^{2} f\right|_{L^{1}} ^{1 / 2},
$$

one gets

$$
\left|T_{\gamma, \lambda, \mu} f\right|_{L^{1}}=\left.\left.|| v\right|^{2} T_{\gamma, \lambda, \mu} f\right|_{L^{1}}=\left|T_{\gamma, \lambda, \mu} f\right|_{L^{p}}=1 .
$$

Besides, Appendix B shows that nonincreasing symmetric rearrangements in $x$ make $J_{N, p}$ decrease:

$$
\forall f \in \mathcal{E}_{p} \quad J_{N, p}\left(f^{* x}\right) \leq J_{N, p}(f)
$$


We deduce that, with no loss of generality, the minimizing sequence can be chosen with the following properties:

$$
\left|f_{n}\right|_{L^{1}}=\left.\left.|| v\right|^{2} f_{n}\right|_{L^{1}}=\left|f_{n}\right|_{L^{p}}=1, \quad f_{n}=f_{n}(|x|, v) .
$$

This sequence fulfills the assumptions of the compactness Lemma D.1 in Appendix D. Hence, up to a subsequence, there exists $f \in \mathcal{E}_{p}$ such that

$$
\begin{gathered}
f_{n} \rightarrow f \text { in } L^{p} \text { if } p<+\infty \text { and } f_{n} \rightarrow f \text { in } L^{\infty} \text { weak } \star \text { if } p=+\infty, \\
E_{f_{n}} \rightarrow E_{f} \text { in } L^{2} .
\end{gathered}
$$

Since we have

$$
\left|E_{f}\right|_{L^{2}}=\lim _{n \rightarrow \infty}\left|E_{f_{n}}\right|_{L^{2}}=\lim _{n \rightarrow \infty}\left(J_{N, p}\left(f_{n}\right)\right)^{-1 / 2}=\left(J_{N, p}\right)^{-1 / 2} \neq 0,
$$

it is clear that $f \neq 0$. Moreover, from the weak convergence of $f_{n}$ in $L^{p}$ and Fatou's lemma, we deduce that

$$
|f|_{L^{1}} \leq 1,\left.\left.\quad|| v\right|^{2} f_{n}\right|_{L^{1}} \leq 1, \quad\left|f_{n}\right|_{L^{p}} \leq 1
$$

and it follows that

$$
J_{N, p}(f) \leq \frac{1}{\left|E_{f}\right|_{L^{2}}^{2}}=J_{N, p}
$$

Since $J_{N, p}=\inf J_{N, p}$, this inequality has to be saturated, as well as the inequalities in $(2.2)$ :

$$
J_{N, p}(f)=\min _{g \in \mathcal{E}_{p} \backslash\{0\}} J_{N, p}(g), \quad|f|_{L^{1}}=\left.\left.|| v\right|^{2} f\right|_{L^{1}}=|f|_{L^{p}}=1 .
$$

Finally, for $p<+\infty$, this enables us to show the strong convergence of the sequence. Indeed, together with the weak $L^{p}$ convergence of $f_{n}$ to $f$, the property $|f|_{L^{p}}=\lim \left|f_{n}\right|_{L^{p}}$ implies that this convergence holds in fact in the $L^{p}$ strong topology. Furthermore, since $\left.\left.|| v\right|^{\alpha} f\right|_{L^{1}}=\left.\left.\lim || v\right|^{\alpha} f_{n}\right|_{L^{1}}$ for $\alpha=0$ or 2 , one can also conclude that $f_{n}$ converges to $f$ and $|v|^{2} f_{n}$ converges to $|v|^{2} f$ in the $L^{1}$ strong topology.

\subsection{Characterization of the minimizers in the case $p<+\infty$}

Thanks to Lemma B.1, one can see that if $Q$ is a minimizer of the functional $J_{N, p}$, then necessarily there exists $x_{0} \in \mathbb{R}^{N}$ such that $\rho_{Q}\left(x-x_{0}\right)$ is spherically symmetric. Furthermore, as remarked above, the functional $J_{N, p}$ is left invariant by the rescaling

$$
f(x, v) \mapsto\left(T_{\gamma, \lambda, \mu} f\right)(x, v)=\gamma f\left(\frac{x}{\lambda}, \mu v\right) .
$$

Let $\left(a_{1}, a_{2}, a_{3}\right)$ be three fixed positive real numbers, that will be precised further. It is readily seen that, for any $f \in \mathcal{E}_{p} \backslash\{0\}$, there exists a unique $(\gamma, \lambda, \mu) \in\left(\mathbb{R}_{+}^{*}\right)^{3}$ such that

$$
\left.\left.|| v\right|^{2} T_{\gamma, \lambda, \mu} f\right|_{L^{1}}=a_{1}, \quad\left|T_{\gamma, \lambda, \mu} f\right|_{L^{1}}=a_{2}, \quad\left|T_{\gamma, \lambda, \mu} f\right|_{L^{p}}=a_{3} .
$$


Therefore, in order to describe the set of all the minimizers of $J_{N, p}$, it is sufficient to characterize all the minimizers $Q$ satisfying

$$
Q \in \mathcal{E}_{p},\left.\left.\quad|| v\right|^{2} Q\right|_{L^{1}}=a_{1}, \quad|Q|_{L^{1}}=a_{2}, \quad|Q|_{L^{p}}=a_{3}, \quad \rho_{Q}=\rho_{Q}(|x|) .
$$

Let $Q$ be such a minimizer of $J_{N, p}$. In order to apply the Euler-Lagrange technique (see $[16]$, [19]), a difficulty comes from the fact that $\mathcal{E}_{p}$ is not an open set, and it is not possible to differentiate the functional $J_{N, p}$ in an arbitrary direction. For any $\varepsilon>0$, let us introduce the set

$$
S_{\varepsilon}=\{(x, v): \quad Q(x, v) \geq \varepsilon\}
$$

(uniquely defined, up to a set of measure zero) and consider a function $g \in L^{\infty}\left(\mathbb{R}^{2 N}\right)$, compactly supported on $\mathbb{R}^{2 N}$ and such that $g \geq 0$ a.e. on $\mathbb{R}^{2 N} \backslash S_{\varepsilon}$. For any $t \in\left(0, \frac{\varepsilon}{|g|_{L^{\infty}}}\right)$ the function $Q+t g$ belongs to $\mathcal{E}_{p} \backslash\{0\}$, which means that

$$
J_{N, p}(Q+t g)-J_{N, p}(Q) \geq 0 .
$$

This implies that the half Gâteaux derivative of $\log J_{N, p}$ at $Q$ in the direction $g$-which is well-defined with the above choice of $g$ - is nonnegative:

$$
\begin{aligned}
0 \leq \lim _{t \rightarrow 0+} \frac{\log J_{N, p}(Q+t g)-\log J_{N, p}(Q)}{t}= & \alpha_{1} \frac{\int|v|^{2} g d x d v}{\int|v|^{2} Q d x d v}+\alpha_{2} \frac{\int g d x d v}{\int Q d x d v} \\
& +\alpha_{3} \frac{\int g Q^{p-1} d x d v}{\int Q^{p} d x d v}+2 \frac{\int \phi_{Q} g d x d v}{\left|E_{Q}\right|_{L^{2}}^{2}}
\end{aligned}
$$

where $\left(\alpha_{1}, \alpha_{2}, \alpha_{3}\right)$ are given by (2.1). Remark that the Poisson equation was used to differentiate the potential energy in the direction $g$ :

$$
\lim _{t \rightarrow 0} \frac{\left|E_{Q+t g}\right|_{L^{2}}^{2}-\left|E_{Q}\right|_{L^{2}}^{2}}{t}=2 \int \nabla_{x} \phi_{Q} \cdot \nabla_{x} \phi_{g} d x=-2 \int \phi_{Q} g d x d v .
$$

With the normalization (2.3) of $Q$, this inequality can be rewritten

$$
\int\left(\frac{\alpha_{1}}{a_{1}}|v|^{2}+\frac{\alpha_{2}}{a_{2}}+\frac{\alpha_{3}}{a_{3}} Q^{p-1}+\frac{2 J_{N, p}}{a_{1}^{\alpha_{1}} a_{2}^{\alpha_{2}} a_{3}^{\alpha_{3}}} \phi_{Q}\right) g d x d v \geq 0 .
$$

Let us now fix $a_{1}, a_{2}, a_{3}$ such that

$$
2 \frac{\alpha_{1}}{a_{1}}=\frac{\alpha_{2}}{a_{2}}=\frac{\alpha_{3}}{a_{3}}=\frac{2 J_{N, p}}{a_{1}^{\alpha_{1}} a_{2}^{\alpha_{2}} a_{3}^{\alpha_{3}}}
$$

(straightforward calculations show that this is always possible, and that the $a_{i}$ 's are uniquely determined by these conditions). Inequality (2.4) becomes

$$
\int\left(Q^{p-1}+1+\frac{|v|^{2}}{2}+\phi_{Q}\right) g d x d v \geq 0
$$


Since $g$ can be chosen with an arbitrary sign on $S_{\varepsilon}$, this means that

$$
Q^{p-1}(x, v)+1+\frac{|v|^{2}}{2}+\phi_{Q}(x) \equiv 0
$$

on $S_{\varepsilon}$ for any $\varepsilon>0$, thus on the support of $Q$. On $\mathbb{R}^{2 N} \backslash S_{\varepsilon}, g$ must be chosen nonnegative and thus from (2.5),

$$
Q^{p-1}(x, v)+1+\frac{|v|^{2}}{2}+\phi_{Q}(x) \geq 0 \quad \text { on } \mathbb{R}^{2 N} \backslash \operatorname{supp}(Q) .
$$

This implies that

$$
Q(x, v)= \begin{cases}\left(-1-\frac{|v|^{2}}{2}-\phi_{Q}(x)\right)^{\frac{1}{p-1}} & \text { if } \frac{|v|^{2}}{2}+\phi_{Q}(x)<-1 \\ 0 & \text { elsewhere }\end{cases}
$$

This is the form (1.17) of the polytropes. It remains to show that there exists a unique spherically symmetric function of this form satisfying the Poisson equation. An integration with respect to $v$ gives

$$
\rho_{Q}(x)=\gamma_{N, p}\left(-1-\phi_{Q}(x)\right)_{+}^{\frac{1}{p-1}+\frac{N}{2}}
$$

where the coefficient $\gamma_{N, p}$ was defined in (1.16). Since, by assumption, $\phi_{Q}$ is spherically symmetric, the Poisson equation is satisfied when this function $\phi_{Q}=\phi_{Q}(r)$ satisfies the ordinary differential equation

$$
\frac{1}{r^{N-1}}\left(r^{N-1} \phi_{Q}^{\prime}\right)^{\prime}=\gamma_{N, p}\left(-1-\phi_{Q}\right)_{+}^{\frac{1}{p-1}+\frac{N}{2}}, \quad \phi_{Q}(r) \rightarrow 0 \text { as } r \rightarrow+\infty .
$$

Uniqueness is now straightforward from the scaling invariance of this equation.

\subsection{Characterization of the minimizers in the case $p=+\infty$}

The above proof in the case $p=+\infty$ requires several adaptations. Inspired by [11], we split it in two steps.

Step $1|Q|_{L^{\infty}}$ is saturated.

We shall first prove that

$$
Q \equiv 1 \text { on } \operatorname{supp}(Q) \text {. }
$$

To this aim, let us modify the set $S_{\varepsilon}$ as follows:

$$
S_{\varepsilon}=\left\{(x, v): \quad \varepsilon \leq Q(x, v) \leq|Q|_{L^{\infty}}-\varepsilon\right\} .
$$


The direction of derivation $g \in L^{\infty}\left(\mathbb{R}^{2 N}\right)$ is now chosen compactly supported on $\mathbb{R}^{2 N}$ and such that

$$
\begin{cases}g \geq 0 & \text { a.e. on } \mathbb{R}^{2 N} \backslash \operatorname{supp}(Q) \\ g=0 & \text { a.e. on }\left\{(x, v): Q(x, v) \geq|Q|_{L^{\infty}}-\varepsilon\right\}\end{cases}
$$

For $t \in\left(0, \frac{\varepsilon}{|g|_{L^{\infty}}}\right)$ we have

$$
0 \leq(Q+t g)(x, v) \leq|Q|_{L^{\infty}} \quad \text { a.e. on } \mathbb{R}^{2 N}
$$

On $\mathcal{E}_{\infty} \backslash\{0\}$, consider the functional

$$
\widetilde{J}_{N, \infty}(f)=\frac{\left.\left.|| v\right|^{2} f\right|_{L^{1}} ^{\alpha_{1}}|f|_{L^{1}}^{\alpha_{2}}}{\left|E_{f}\right|_{L^{2}}^{2}}
$$

From (2.7), one can deduce that, for $t>0$ small enough, $Q+\operatorname{tg} \in \mathcal{E}_{\infty} \backslash\{0\}$ and

$$
\widetilde{J}_{N, \infty}(Q+t g)=\frac{1}{|Q+t g|_{L^{\infty}}^{\alpha_{3}}} J_{N, \infty}(Q+t g) \geq \frac{1}{|Q|_{L^{\infty}}^{\alpha_{3}}} J_{N, \infty}=\widetilde{J}_{N, \infty}(Q) .
$$

Therefore

$$
\begin{aligned}
0 \leq \lim _{t \rightarrow 0+} \frac{\log \widetilde{J}_{N, \infty}(Q+t g)-\log \widetilde{J}_{N, \infty}(Q)}{t} & =\alpha_{1} \frac{\int|v|^{2} g d x d v}{\int|v|^{2} Q d x d v}+\alpha_{2} \frac{\int g d x d v}{\int Q d x d v}+2 \frac{\int \phi_{Q} g d x d v}{\left|E_{Q}\right|_{2}^{L^{2}}} \\
& =\int\left(\frac{\alpha_{1}}{a_{1}}|v|^{2}+\frac{\alpha_{2}}{a_{2}}+\frac{2 J_{N, \infty}}{a_{1}^{\alpha_{1}} a_{2}^{\alpha_{2}} a_{3}^{\alpha_{3}}} \phi_{Q}\right) g d x d v .
\end{aligned}
$$

By fixing $a_{1}, a_{2}, a_{3}$ such that

$$
a_{3}=|Q|_{L^{\infty}}=1, \quad 2 \frac{\alpha_{1}}{a_{1}}=\frac{\alpha_{2}}{a_{2}}=\frac{2 J_{N, \infty}}{a_{1}^{\alpha_{1}} a_{2}^{\alpha_{2}} a_{3}^{\alpha_{3}}}
$$

we get

$$
\int\left(1+\frac{|v|^{2}}{2}+\phi_{Q}(x)\right) g d x d v \geq 0
$$

for any suitable $g$. Since $g$ has an arbitrary sign on $S_{\varepsilon}$ and since

$$
\text { meas }\left\{(x, v): 1+\frac{|v|^{2}}{2}+\phi_{Q}(x)=0\right\}=0,
$$

we infer from (2.8) that

$$
\text { meas }\{(x, v): 0<Q(x, v)<1\}=0
$$

and thus (2.6) holds. Let $Q^{* v}$ be the nonincreasing symmetrical rearrangement of $Q$ with respect to the variable $v$ ( $x$ being a parameter). This operation does not modify the $L^{1}$ 
and $L^{\infty}$ norms of $Q$, nor the corresponding potential energy. Furthermore, the function $|v| \mapsto|v|^{2}$ being strictly increasing, Lemma B.1 shows that

$$
\int|v|^{2} Q^{* v} d x d v \leq \int|v|^{2} Q d x d v \quad \text { and } \quad J_{N, \infty}\left(Q^{* v}\right) \leq J_{N, \infty}(Q)
$$

with strict inequalities unless $Q^{* v}=Q$ a.e. Since $Q$ is a minimizer of $J_{N, \infty}$, this is enough to conclude that $Q^{* v}=Q$ ie $Q(x, v)=Q(x,|v|)$ is decreasing with respect to $v$. Since $Q$ takes only the values 0 or 1 , a simple integration in $v$ yields:

$$
Q(x, v)= \begin{cases}1 & \text { if }|v| \leq\left(\frac{\rho_{Q}(x)}{\omega_{N}}\right)^{1 / N} \\ 0 & \text { elsewhere. }\end{cases}
$$

Step 2 A variational problem for the density $\rho$.

We are now able to reformulate our problem as a minimization problem for the unknown $\rho_{Q}$. For any $\rho \in L^{1} \cap L^{(N+2) / N}\left(\mathbb{R}^{N}\right)$, we define $f[\rho]$ by

$$
f[\rho](x, v)= \begin{cases}1 & \text { if }|v| \leq\left(\frac{\rho(x)}{\omega_{N}}\right)^{1 / N} \\ 0 & \text { elsewhere. }\end{cases}
$$

For $\alpha=0$ or 2 , direct calculations give

$$
\left.\left.|| v\right|^{\alpha} f[\rho]\right|_{L^{1}}=\frac{N}{N+\alpha}\left(\omega_{N}\right)^{-\alpha / N}\left(|\rho|_{L^{(N+\alpha) / N}}\right)^{(N+\alpha) / N}
$$

so one can check that $f[\rho]$ belongs to $\mathcal{E}_{\infty}$. If $\rho \neq 0$, consider the functional

$$
G_{N}(\rho)=C_{0} \frac{|\rho|_{L^{(N+2) / N}}^{\beta_{1}}|\rho|_{L^{1}}^{\beta_{2}}}{\left|E_{f[\rho]}\right|_{L^{2}}^{2}}
$$

where

$$
\beta_{1}=\alpha_{1} \frac{N+2}{N}, \quad \beta_{2}=\alpha_{2}, \quad C_{0}=\left(\frac{N}{N+2}\left(\omega_{N}\right)^{-2 / N}\right)^{-\alpha_{1}},
$$

then from (2.10),

$$
G_{N}(\rho)=J_{N, \infty}(f[\rho]) \geq J_{N, \infty}
$$

Now since from (2.9) $Q=f\left[\rho_{Q}\right]$, we conclude

$$
G_{N}\left(\rho_{Q}\right)=J_{N, \infty}=\min _{\rho \in L^{1} \cap L^{(N+2) / N} \backslash\{0\}} G_{N}(\rho) .
$$

$\rho_{Q}$ is a minimizer of this functional $G_{N}$, which is spherically symmetric in $x$ and such that

$$
\left|\rho_{Q}\right|_{L^{(N+2) / N}}=\frac{N+2}{N} \omega_{N}^{2 / N} a_{1}, \quad\left|\rho_{Q}\right|_{L^{1}}=a_{2} .
$$


By proceeding as for the characterization of $Q$ in the case $p<+\infty$, one can compute the Euler-Lagrange equation for $G_{N}$ (we skip the details of the computations) and obtain

$$
\rho_{Q}(x)=2^{N / 2} \omega_{N}\left(-1-\phi_{Q}(x)\right)_{+}^{N / 2} .
$$

By plugging this formula into the Poisson equation, we obtain the ordinary differential equation satisfied by the potential $\phi_{Q}$ :

$$
\frac{1}{r^{N-1}}\left(r^{N-1} \phi_{Q}^{\prime}\right)^{\prime}=2^{N / 2} \omega_{N}\left(-1-\phi_{Q}\right)_{+}^{N / 2} \quad \phi_{Q}(r) \rightarrow 0 \text { as } r \rightarrow+\infty,
$$

the scaling properties of this ODE ensuring the uniqueness of $\phi_{Q}$. Remark that from (2.9) and (2.11), we recover the form (1.17) of the polytrope for $p=\infty$ and the corresponding constant $\gamma_{p, \infty}=2^{N / 2} \omega_{N}$ given by $(1.16)$.

\section{The concentration compactness argument}

This section is devoted to the key Lemmas at the heart of the non linear stability results of $Q_{p}$ in dimension $N=3,4$, namely Theorem 1.4, Proposition 1.11 and Theorem 1.12. We first claim a simple modification of the standard concentration compactness Lemma and then study the behavior of the potential energy in each regime. Note that remarkably enough, up to some slight modifications, most estimates we will need are already contained in [24], section II, and we should follow the same lines.

We work in the whole section in dimension $N=3,4$ and note $L_{+}^{1}\left(\mathbb{R}^{2 N}\right)=\{f \geq$ 0 with $\left.f \in L^{1}\left(\mathbb{R}^{2 N}\right)\right\}$. We claim the following Lemma which is an adaptation of the standard concentration compactness Lemma, i.e. Lemma 1.1 in [24].

Lemma 3.1 (Concentration compactness Lemma) Let $M>0$ and $\left(f_{n}\right)_{n \geq 1}$ a sequence in $L_{+}^{1}\left(\mathbb{R}^{2 N}\right)$. Let $\rho_{n}(x)=\int f_{n}(x, v) d v$ and assume

$$
\int_{\mathbb{R}^{N}} \rho_{n}(x) d x=M \text { and } \sup _{n} \iint|v|^{2} f_{n}(x, v) d v d x<+\infty .
$$

Then there exists a subsequence $\left(f_{n_{k}}\right)_{k \geq 1}$ such that one of the following three possibilities occurs:

(i) Compactness: there exists $y_{k} \in \mathbb{R}^{N}$ such that

$$
\forall \varepsilon>0, \quad \exists R<+\infty \quad \text { such that for all } k \geq 1 \quad \int_{y_{k}+B_{R}} \rho_{n_{k}}(x) d x \geq M-\varepsilon
$$

(ii) Vanishing:

$$
\forall R<+\infty, \quad \lim _{k \rightarrow+\infty} \sup _{y \in \mathbb{R}^{N}} \int_{y+B_{R}} \rho_{n_{k}}(x) d x=0 ;
$$


(iii) Dichotomy: there exists $m \in(0, M)$ such that for all $\varepsilon>0$, there exists $k_{0} \geq 1$ and $f_{k}^{1}, f_{k}^{2} \in L_{+}^{1}\left(\mathbb{R}^{2 N}\right)$ such that for all $k \geq k_{0}$,

$$
\left\{\begin{array}{l}
f_{n_{k}}=f_{k}^{1}+f_{k}^{2}+v_{k} \quad \text { with } 0 \leq f_{k}^{1}, f_{k}^{2}, v_{k} \leq f_{n_{k}}, \quad f_{k}^{1} f_{k}^{2}=f_{k}^{1} v_{k}=f_{k}^{2} v_{k}=0 \quad \text { a.e., } \\
\rho_{n_{k}}=\rho_{k}^{1}+\rho_{k}^{2}+w_{k} \quad \text { with } 0 \leq \rho_{k}^{1}, \rho_{k}^{2}, w_{k} \leq \rho_{n_{k}}, \quad \rho_{k}^{1} \rho_{k}^{2}=\rho_{k}^{1} w_{k}=\rho_{k}^{2} w_{k}=0 \quad \text { a.e., } \\
\operatorname{dist}\left(\operatorname{Supp}\left(f_{k}^{1}, f_{k}^{2}\right)\right) \rightarrow+\infty, \quad \operatorname{dist}\left(\operatorname{Supp}\left(\rho_{k}^{1}, \rho_{k}^{2}\right)\right) \rightarrow+\infty \quad \text { as } \quad k \rightarrow+\infty, \\
\left|\rho_{n_{k}}-\rho_{k}^{1}-\rho_{k}^{2}\right|_{L^{1}} \leq \varepsilon, \quad\left|\int_{\mathbb{R}^{N}} \rho_{k}^{1}(x) d x-m\right|+\left|\int_{\mathbb{R}^{N}} \rho_{k}^{2}(x) d x-(M-m)\right|<\varepsilon .
\end{array}\right.
$$

The proof of Lemma 3.1 is standard and we sketch it for the sake of completeness in Appendix D.

We now study the behavior of the potential energy in each regime and have the following Lemma at the heart of the use of these techniques for variational problems.

Lemma 3.2 (Control of the potential energy) Let $\left(f_{n}\right)_{n \geq 1}$ as in Lemma 3.1. Assume moreover that there holds for some $p_{\text {crit }}<p \leq+\infty$,

$$
\limsup _{n \rightarrow+\infty}\left|f_{n}\right|_{L^{p}}<+\infty .
$$

Consider the Poisson force field

$$
E_{f_{n}}(x)=\frac{1}{N \omega_{N}} \frac{x}{|x|^{N}} \star \rho_{n}(x),
$$

then we have the following:

(i) Compactness: if compactness occurs, then up to a subsequence,

$$
\begin{gathered}
f_{n_{k}}\left(\cdot+y_{k}\right) \rightarrow f \text { in the } L^{1} \text { and } L^{p} \text { weak topologies for } p<\infty, \\
f_{n_{k}}\left(\cdot+y_{k}\right) \rightarrow f \text { in } L^{1} \text { weak and in } L^{\infty} \text { weak } \star \text { for } p=+\infty .
\end{gathered}
$$

Moreover, in both cases we have $f \in \mathcal{E}_{p}$ and

$$
\int_{\mathbb{R}^{2 N}} f=M \quad \text { and } \quad E_{f_{n_{k}}\left(\cdot+y_{k}\right)} \rightarrow E_{f} \quad \text { in } \quad L^{2} \quad \text { as } k \rightarrow+\infty .
$$

(ii) Vanishing: if vanishing occurs, then

$$
\left|E_{f_{n_{k}}}\right|_{L^{2}} \rightarrow 0 \text { as } k \rightarrow+\infty .
$$

(iii) Dichotomy: if dichotomy occurs, then

$$
\left.\limsup _{k \rightarrow+\infty}|| E_{f_{n_{k}}}\right|_{L^{2}} ^{2}-\left|E_{f_{k}^{1}}\right|_{L^{2}}^{2}-\left|E_{f_{k}^{2}}\right|_{L^{2}}^{2} \mid<\varepsilon^{\theta},
$$

with $\theta=\frac{4 N+4-N^{2}}{4 N(p-1)}\left(p-p_{\text {crit }}\right)>0$. 


\section{Proof of Lemma 3.2}

Let us say again that all estimates we shall need have somehow already been obtained in [24], section II, and we should follow the same lines. Let

$$
q=\frac{N(p-1)+2 p}{N(p-1)+2} \text { so that } p>p_{\text {crit }} \text { is equivalent to } q>\frac{2 N}{N+2}
$$

and recall the interpolation estimate

$$
|\rho|_{L^{q}} \leq\left.\left. C|f|_{L^{p}}^{\frac{2 p}{p(N+2)-N}}|| v\right|^{2} f\right|_{L^{1}} ^{\frac{N(p-1)}{p(N+2)-N}}
$$

then from (3.1) and (3.5),

$$
\forall r \in[1, q], \quad \limsup _{n \rightarrow+\infty}\left|\rho_{n}\right|_{L^{r}}<+\infty .
$$

Recall also that the potential energy is given by

$$
|E|_{L^{2}}^{2}=C_{0} \iint \frac{\rho(x) \rho(y)}{|x-y|^{N-2}} d x d y,
$$

with $C_{0}=\frac{1}{N(N-2) \omega_{N}}$ (without loss of generality, we skip this constant $C_{0}$ in the sequel of the proof) and controlled according to

$$
|E|_{L^{2}}^{2} \leq\left.\left. C|f|_{L^{1}}^{\frac{4 N+4-N^{2}}{2 N(p-1)}\left(p-p_{c r i t}\right)}|f|_{L^{p}}^{\frac{p(N-2)}{N(p-1)}}|| v\right|^{2} f\right|_{L^{1}} ^{\frac{N-2}{2}}
$$

Step 1 Dichotomy.

Assume that dichotomy, i.e. (3.4), occurs. Pick an $\varepsilon>0$. We let $\rho_{n_{k}}=\rho_{k}^{1}+\rho_{k}^{2}+w_{k}$ as in (3.4) and compute

$$
\begin{aligned}
& \left|E_{f_{n_{k}}}\right|_{L^{2}}^{2}-\left|E_{f_{k}^{1}}\right|_{L^{2}}^{2}-\left|E_{f_{k}^{2}}\right|_{L^{2}}^{2}=2 \int_{R^{2 N}} \frac{\rho_{k}^{1}(x) \rho_{k}^{2}(y)}{|x-y|^{N-2}} d x d y \\
+ & 2 \int_{R^{2 N}} \frac{\rho_{n_{k}}(x) w_{k}(y)}{|x-y|^{N-2}} d x d y-\int_{\mathbb{R}^{2 N}} \frac{w_{k}(x) w_{k}(y)}{|x-y|^{N-2}} d x d y .
\end{aligned}
$$

For the first term, let $d_{k}=\operatorname{dist}\left(\operatorname{Supp}\left(\rho_{k}^{1}, \rho_{k}^{2}\right)\right)$, then

$$
\int_{R^{2 N}} \frac{\rho_{k}^{1}(x) \rho_{k}^{2}(y)}{|x-y|^{N-2}} d x d y \leq \frac{M^{2}}{d_{k}^{N-2}} \rightarrow 0 \quad \text { as } \quad k \rightarrow+\infty,
$$

from (3.4). For the last two terms, we have from the generalized Young inequality

$$
\begin{aligned}
\left|\int_{R^{2 N}} \frac{\rho_{n_{k}}(x) w_{k}(y)}{|x-y|^{N-2}} d x d y\right|+\left|\int_{\mathbb{R}^{2 N}} \frac{w_{k}(x) w_{k}(y)}{|x-y|^{N-2}} d x d y\right| \\
\leq C\left|\rho_{n_{k}}\right|_{L^{\frac{2 N}{N+2}}}\left|w_{k}\right|_{L^{\frac{2 N}{N+2}}}+C\left|w_{k}\right|_{L^{2 N+2}}^{2} .
\end{aligned}
$$


Now from (3.4), (3.11) and (3.12) and by interpolation, we get

$$
\left|w_{k}\right|_{L^{\frac{2 N}{N+2}}} \leq C\left|w_{k}\right|_{L^{1}}^{\theta}<C \varepsilon^{\theta} .
$$

This concludes the proof of (3.10).

Step 2 Vanishing.

Assume that vanishing occurs, i.e. (3.3). Pick an $\varepsilon>0$. We split the convolution kernel in (3.13) in three parts:

$$
\begin{aligned}
\left|E_{f_{n_{k}}}\right|_{L^{2}}^{2} & =\iint_{|x-y|>R} \frac{\rho_{n_{k}}(x) \rho_{n_{k}}(y)}{|x-y|^{N-2}} d x d y+\iint_{|x-y|<\eta} \frac{\rho_{n_{k}}(x) \rho_{n_{k}}(y)}{|x-y|^{N-2}} d x d y \\
& +\iint_{\eta<|x-y|<R} \frac{\rho_{n_{k}}(x) \rho_{n_{k}}(y)}{|x-y|^{N-2}} d x d y .
\end{aligned}
$$

The first term is estimated from $L^{1}$ control on $\rho_{n_{k}}$ :

$$
\iint_{|x-y|>R} \frac{\rho_{n_{k}}(x) \rho_{n_{k}}(y)}{|x-y|^{N-2}} d x d y \leq \frac{M^{2}}{R^{N-2}}<\varepsilon
$$

for $R>0$ large enough. We rewrite the second term as a convolution with the kernel $K_{\eta}(x)=\frac{\mathbf{1}_{|x| \leq \eta}}{|x|^{N-2}}$ and estimate from Young's inequality:

$$
\left|\rho_{n_{k}} K_{\eta} \star \rho_{n_{k}}\right|_{L^{1}} \leq C\left|\rho_{n_{k}}\right|_{L^{q}}^{2}\left|K_{\eta}\right|_{L^{r}} \quad \text { with } \quad r=\frac{1}{2} \frac{q}{q-1} .
$$

Now from (3.11) and (3.12),

$$
r<\frac{N}{N-2} \quad \text { and thus } \quad\left|\rho_{n_{k}}\right|_{L^{q}}^{2}\left|K_{\eta}\right|_{L^{r}} \leq C \eta^{N-r(N-2)}<\varepsilon
$$

for $\eta>0$ small enough. The real numbers $R, \eta>0$ being chosen, we now estimate the last term in (3.15):

$$
\begin{aligned}
\iint_{\eta<|x-y|<R} \frac{\rho_{n_{k}}(x) \rho_{n_{k}}(y)}{|x-y|^{N-2}} d x d y & \leq \frac{1}{\eta^{N-2}} \int \rho_{n_{k}}(y)\left(\int_{|x-y| \leq R} \rho_{n_{k}}(x) d x\right) d y \\
& \leq \frac{M}{\eta^{N-2}} \sup _{y \in \mathbb{R}^{N}} \int_{y+B_{R}} \rho_{n_{k}}(x) d x \longrightarrow 0 \text { as } k \rightarrow+\infty
\end{aligned}
$$

from (3.3). Letting $\varepsilon \rightarrow 0$ concludes the proof of (3.9).

Step 3 Compactness. 
Assume that compactness (3.2) occurs. First observe that the sequence of probability measures $\bar{f}_{n_{k}}(x, v)=f_{n_{k}}\left(x+y_{k}, v\right)$ is tight in $(x, v)$ from (3.2) and the uniform control of the kinetic energy (3.1), that is:

$$
\forall \varepsilon>0, \quad \exists R(\varepsilon) \text { such that } \forall k \geq 1, \quad \int_{Y \in \mathbb{R}^{2 N}, \quad|Y| \geq R(\varepsilon)} \bar{f}_{n_{k}}(X) d X<\varepsilon .
$$

Moreover, no concentration can occur from uniform $L^{p}$ bound (3.5). Thus $\left(\bar{f}_{n_{k}}\right)_{k \geq 1}$ satisfies the hypothesis of the Dunford-Pettis theorem and is weakly relatively compact in $L^{1}$ :

$$
\bar{f}_{n_{k}} \rightarrow f \text { in } L^{1} \text { with } f \in \mathcal{E}_{p} \text { and } \int_{\mathbb{R}^{2 N}} f(X) d X=M,
$$

and the weak convergence also holds in $L^{p}$ for $p<\infty$ (and $L^{\infty}$ weak for $p=\infty$ ). Let us observe that the weak convergence in $L^{1}$ gives

$$
\forall \phi \in L^{\infty}, \quad \int \bar{\rho}_{n_{k}}(x) \phi(x) d x \rightarrow \int \rho(x) \phi(x) d x \quad \text { as } k \rightarrow+\infty .
$$

To prove the convergence of the potential energy, we observe that

$$
\left|E_{\bar{f}_{n_{k}}}-E_{f}\right|_{L^{2}}^{2}=\int_{\mathbb{R}^{N} \times \mathbb{R}^{N}} \frac{\left(\rho(x)-\bar{\rho}_{n_{k}}(x)\right)\left(\rho(y)-\bar{\rho}_{n_{k}}(y)\right)}{|x-y|^{N-2}} d x d y .
$$

Pick an $\varepsilon>0$. We split the kernel as in the vanishing case and estimate

$$
\begin{aligned}
& \mid \int_{\mathbb{R}^{N} \times \mathbb{R}^{N}} \frac{\left(\rho(x)-\bar{\rho}_{n_{k}}(x)\right)\left(\rho(y)-\bar{\rho}_{n_{k}}(y)\right)}{|x-y|^{N-2}} d x d y \mid \leq C \eta^{N-r(N-2)} \\
&+\frac{C}{R^{N-2}}+\left|\iint_{\eta<|x-y|<R} \frac{\left(\rho(x)-\bar{\rho}_{n_{k}}(x)\right)\left(\rho(y)-\bar{\rho}_{n_{k}}(y)\right)}{|x-y|^{N-2}} d x d y\right| .
\end{aligned}
$$

Let

$$
v_{n_{k}}(x)=\int_{\mathbb{R}^{N}}\left(\bar{\rho}_{n_{k}}(y)-\rho(y)\right) h_{R}^{\eta}(|x-y|) d y, \quad \text { with } \quad h_{R}^{\eta}(x)=\frac{\mathbf{1}_{\eta<|x|<R}}{|x|^{N-2}} .
$$

Then $v_{n_{k}}$ is uniformly bounded in $L^{1} \cap L^{\infty}$ and from $h_{R}^{\eta} \in L^{\infty}$ and (3.17),

$$
v_{n_{k}}(x) \rightarrow 0 \quad \forall x \in \mathbb{R}^{N},
$$

and thus $v_{n_{k}} \rightarrow 0$ in $L_{l o c}^{1}$. Moreover, there is no mass loss from (3.16), and thus:

$$
\begin{aligned}
\int_{\mathbb{R}^{2 N}} \bar{\rho}_{n_{k}}(y) h_{R}^{\eta}(|x-y|) d x d y & =\left(\int_{\mathbb{R}^{N}} h_{R}^{\eta} d x\right)\left(\int_{\mathbb{R}^{N}} \bar{\rho}_{n_{k}}\right) \\
& =\left(\int_{\mathbb{R}^{N}} h_{R}^{\eta} d x\right)\left(\int_{\mathbb{R}^{N}} \rho\right)=\int_{\mathbb{R}^{2 N}} \rho(y) h_{R}^{\eta}(|x-y|) d x d y .
\end{aligned}
$$


This implies that in fact $v_{n_{k}} \rightarrow 0$ in $L^{1}$ as $k \rightarrow+\infty$, and we have also

$$
v_{n_{k}} \rightarrow 0 \text { in } L^{p} \text { for } 1 \leq p<+\infty .
$$

We deduce from (3.12) that

$$
\int_{\mathbb{R}^{N}} \bar{\rho}_{n_{k}} v_{n_{k}} \rightarrow 0 \text { as } k \rightarrow+\infty
$$

and thus

$$
\int_{\mathbb{R}^{N} \times \mathbb{R}^{N}} \frac{\left(\rho(x)-\bar{\rho}_{n_{k}}(x)\right)\left(\rho(y)-\bar{\rho}_{n_{k}}(y)\right)}{|x-y|^{N-2}} d x d y \rightarrow 0 \text { as } k \rightarrow+\infty .
$$

This concludes the proof of $L^{2}$ convergence (3.8). The proof of Lemma 3.2 is complete.

\section{Proof of the stability result for $N=3$}

This section is devoted to the proof of the nonlinear stability of $Q_{p}$ in dimension $N=3$. In the first subsection, we obtain some preliminary results concerning the minimization problem (1.23), while the second and the third subsections are respectively devoted to the proofs of Theorem 1.4 and Corollary 1.6.

\subsection{Preliminary results}

Let a convex function $j$ as in the hypothesis of Theorem 1.4. Recall that for $M_{1}, M_{j}>0$, we have set

$$
\mathcal{F}\left(M_{1}, M_{j}\right)=\left\{f \in \mathcal{E}_{p} \text { with } \int_{\mathbb{R}^{6}} f=M_{1}, \quad \int_{\mathbb{R}^{6}} j(f)=M_{j}\right\}
$$

and

$$
I\left(M_{1}, M_{j}\right)=\inf _{f \in \mathcal{F}\left(M_{1}, M_{j}\right)} \mathcal{H}(f)
$$

Our aim in this subsection is to collect some preliminary informations on the minimization problem (1.23) and then to prove Lemma 1.5.

Lemma 4.1 (Preliminary properties for the minimization problem (1.23)) We have

$$
-\infty<I\left(M_{1}, M_{j}\right)<0 .
$$

Moreover, for all $0<\alpha<1$,

$$
\begin{gathered}
I\left(M_{1}, \alpha M_{j}\right) \geq I\left(M_{1}, M_{j}\right), \\
I\left(\alpha M_{1}, \alpha M_{j}\right)=\alpha^{\frac{7}{3}} I\left(M_{1}, M_{j}\right) .
\end{gathered}
$$




\section{Proof of Lemma 4.1}

Given $M_{1}, M_{j}>0$ we first claim that $\mathcal{F}\left(M_{1}, M_{j}\right)$ is non empty. Indeed, let the rescaling

$$
\tilde{f}(x, v)=\frac{\mu}{\lambda^{2}} f\left(\frac{x}{\lambda}, \mu v\right),
$$

we compute from the formulae of Appendix A:

$$
\mathcal{H}(\tilde{f})=a^{3} r^{7} \mathcal{H}(f), \quad|\tilde{f}|_{L^{1}}=a r^{3}|f|_{L^{1}}, \quad|j(\tilde{f})|_{L^{1}}=r^{3}|j(a f)|_{L^{1}}
$$

with

$$
a=\frac{\mu}{\lambda^{2}} \text { and } r=\frac{\lambda}{\mu} .
$$

Pick a non zero $f$ in $\mathcal{E}_{p}$ such that $j(f) \in L^{1}$ and let $(r, a)$ be such that

$$
a r^{3}|f|_{L^{1}}=M_{1}=|\tilde{f}|_{L^{1}}
$$

We then have

$$
|j(\tilde{f})|_{L^{1}}=\frac{M_{1}}{|f|_{L^{1}}}|j(f)|_{L^{1}} h(a, f)
$$

with

$$
h(a, f)=\frac{|j(a f)|_{L^{1}}}{a|j(f)|_{L^{1}}} .
$$

Now from $(1.22), h(a, f)$ is a continuous function of $a$ with

$$
h(0, f)=0 \text { and } h(a, f) \rightarrow+\infty \text { as } a \rightarrow+\infty,
$$

and thus there exists $a>0$ such that $|j(\tilde{f})|_{L^{1}}=M_{j}$.

To prove (4.1), we first observe that by (1.5) and (1.22):

$$
\forall f \in \mathcal{F}\left(M_{1}, M_{j}\right) \quad|E|_{L^{2}}^{2} \leq\left.\left. C_{M_{1}, M_{j}}|| v\right|^{2} f\right|^{1 / 2}
$$

and thus $I\left(M_{1}, M_{j}\right)>-\infty$. Next, for any $f \in \mathcal{F}\left(M_{1}, M_{j}\right)$, we rescale this function as follows:

$$
\hat{f}(x, v)=f\left(\frac{x}{\lambda}, \lambda v\right)
$$

so that

$$
|\hat{f}|_{L^{1}}=|f|_{L^{1}}, \quad|j(\hat{f})|_{L^{1}}=|j(f)|_{L^{1}}, \quad \mathcal{H}(\hat{f})=\left.\left.\frac{1}{\lambda^{2}}|| v\right|^{2} f\right|_{L^{1}}-\frac{1}{\lambda}\left|E_{f}\right|_{L^{2}}^{2}
$$

Now the infimum of $a x^{2}-b x$ is $-\frac{b^{2}}{2 a}<0$, and (4.1) follows.

We now turn to the proof of (4.2). From (4.1), there is a non zero $f$ with

$$
\mathcal{H}(f)<0 \text {. }
$$


We rescale $f$ as in (4.4) to reach $|\tilde{f}|_{L^{1}}=M_{1},|j(\tilde{f})|_{L^{1}}=\alpha|j(f)|_{L^{1}}$ i.e.

$$
a r^{3}=1, \quad r^{3}|j(a f)|_{L^{1}}=\alpha|j(f)|_{L^{1}} .
$$

We thus have

$$
h(a, f)=\alpha<1
$$

and from $h(1, f)=1$ and (4.6), we may solve the $(a, r)$ system with $a<1$. We conclude

$$
\mathcal{H}(\tilde{f})=a^{3} r^{7} \mathcal{H}(f)=a^{\frac{2}{3}} \mathcal{H}(f)>\mathcal{H}(f)
$$

from (4.7), and (4.2) follows.

It remains to prove (4.3). For any $f \in \mathcal{F}\left(M_{1}, M_{j}\right)$, one obtains from (4.4) a function $\tilde{f} \in \mathcal{F}\left(\alpha M_{1}, \alpha M_{j}\right)$ by setting $a=\frac{\mu}{\lambda^{2}}=1$ and $r^{3}=\alpha$. We then compute

$$
\mathcal{H}(\tilde{f})=a^{3} r^{7} \mathcal{H}(f)=\alpha^{\frac{7}{3}} \mathcal{H}(f)
$$

and (4.3) follows. This concludes the proof of Lemma 4.1.

\section{Proof of Lemma 1.5}

Assume first that $j(f)=f^{p}$ with $\frac{9}{7}<p<+\infty$. By using the rescaling (4.4), we explicitly compute:

$$
\forall \alpha, \beta>0, \quad I\left(\alpha M_{1}, \beta M_{j}\right)=\alpha^{\frac{7 p-9}{3(p-1)}} \beta^{\frac{2}{3(p-1)}} I\left(M_{1}, M_{j}\right)
$$

and thus from (4.1), (1.24) is equivalent to: $\forall 0<\alpha<1, \forall 0 \leq \beta \leq 1$,

$$
\alpha^{\frac{7 p-9}{3(p-1)}} \beta^{\frac{2}{3(p-1)}}+(1-\alpha)^{\frac{7 p-9}{3(p-1)}}(1-\beta)^{\frac{2}{3(p-1)}}<1 .
$$

The cases $\beta=0$ or $\beta=1$ are straighforward. Assume thus $0<\beta<1$ and observe that

$$
\frac{7 p-9}{3(p-1)}+\frac{2}{3(p-1)}=\frac{7}{3}>2
$$

so that one of the two exponents must be strictly greater than one. We then conclude using the following standard convexity statement:

$$
\forall b>1, \quad \forall 0<x<1, \quad x^{b}+(1-x)^{b}<1,
$$

and (4.9) follows.

Assume now that $j$ satisfies the homogeneity estimate (1.26):

$$
\forall t \geq 0, \quad \forall b \geq 1, \quad b^{p_{1}} j(t) \leq j(b t) \leq b^{p_{2}} j(t)
$$

for some $\frac{3}{2}<p_{1} \leq p_{2}<+\infty$. We claim that for any $0 \leq \lambda \leq 1$,

$$
I\left(\lambda M_{1}, M_{j}\right) \geq \lambda^{\frac{7 p_{1}-9}{3\left(p_{1}-1\right)}} I\left(M_{1}, M_{j}\right)
$$




$$
I\left(M_{1}, \lambda M_{j}\right) \geq \lambda^{\frac{2}{3\left(p_{2}-1\right)}} I\left(M_{1}, M_{j}\right) .
$$

To prove (4.11), we use again the rescaling (4.4) in order to reach $|\tilde{f}|_{L^{1}}=\lambda|f|_{L^{1}},|j(\tilde{f})|_{L^{1}}=$ $|j(f)|_{L^{1}}$. This means that $a r^{3}=\lambda$ and $h(a, f) a r^{3}=1$ with $h$ given by (4.5). Hence $h(a, f)=\frac{1}{\lambda}>1$ and we may solve the $(a, r)$ system with $a>1$. From (1.26), we then obtain the estimate $j(a f) \geq a^{p_{1}} j(f)$ and thus

$$
a^{p_{1}-1} \leq \frac{|j(a f)|_{L^{1}}}{a|j(f)|_{L^{1}}}=h(a, f)=\frac{1}{\lambda} .
$$

Computing the Hamiltonian yields

$$
\mathcal{H}(\tilde{f})=a^{3} r^{7} \mathcal{H}(f)=a^{\frac{2}{3}} \lambda^{\frac{7}{3}} \mathcal{H}(f) \geq \lambda^{\frac{7 p_{1}-9}{3\left(p_{1}-1\right)}} \mathcal{H}(f)
$$

if we chose $\mathcal{H}(f)<0$ from (4.1), and (4.11) follows.

To prove (4.12), we use the rescaling (4.4) to reach $|\tilde{f}|_{L^{1}}=|f|_{L^{1}},|j(\tilde{f})|_{L^{1}}=\lambda|j(f)|_{L^{1}}$. Hence we have $a r^{3}=1, h(a, f) a r^{3}=\lambda$ and we deduce that $h(a, f)=\lambda<1$, thus we may choose $a<1$. Then, from (1.26) we obtain the estimate $j(a f) \geq a^{p_{2}} j(f)$ and thus

$$
a^{p_{2}-1} \leq h(a, f)=\lambda .
$$

This yields

$$
\mathcal{H}(\tilde{f})=a^{3} r^{7} \mathcal{H}(f)=a^{\frac{2}{3}} \mathcal{H}(f) \geq \lambda^{\frac{2}{3\left(p_{2}-1\right)}} \mathcal{H}(f)
$$

for $\mathcal{H}(f)<0$ and (4.12) follows.

Let now $0<\alpha<1,0 \leq \beta \leq 1$, we need to prove (1.24). By possibly exchanging $\alpha$ for $1-\alpha$ and $\beta$ for $1-\beta$, we may assume $\beta \leq \alpha$, i.e.

$$
\frac{\beta}{\alpha} \leq 1, \quad \frac{1-\alpha}{1-\beta} \leq 1 .
$$

We then estimate from (4.3) and (4.12):

$I\left(\alpha M_{1}, \beta M_{j}\right)=\alpha^{\frac{7}{3}} I\left(M_{1}, \frac{\beta}{\alpha} M_{j}\right) \geq \alpha^{\frac{7}{3}}\left(\frac{\beta}{\alpha}\right)^{\frac{2}{3\left(p_{2}-1\right)}} I\left(M_{1}, M_{j}\right)=\alpha^{\frac{7 p_{2}-9}{3\left(p_{2}-1\right)}} \beta^{\frac{2}{3\left(p_{2}-1\right)}} I\left(M_{1}, M_{j}\right)$, and, similarly, from (4.3) and (4.11):

$$
\begin{gathered}
I\left((1-\alpha) M_{1},(1-\beta) M_{j}\right)=(1-\beta)^{\frac{7}{3}} I\left(\frac{1-\alpha}{1-\beta} M_{1}, M_{j}\right) \\
\geq(1-\beta)^{\frac{7}{3}}\left(\frac{1-\alpha}{1-\beta}\right)^{\frac{7 p_{1}-9}{3\left(p_{1}-1\right)}} I\left(M_{1}, M_{j}\right)=(1-\alpha)^{\frac{7 p_{1}-9}{3\left(p_{1}-1\right)}}(1-\beta)^{\frac{2}{3\left(p_{1}-1\right)}} I\left(M_{1}, M_{j}\right) .
\end{gathered}
$$

We now observe that $\frac{3}{2}<p_{1} \leq p_{2}$ implies

$$
1<\frac{7 p_{1}-9}{3\left(p_{1}-1\right)} \leq \frac{7 p_{2}-9}{3\left(p_{2}-1\right)}
$$


and thus

$$
\alpha^{\frac{7 p_{2}-9}{3\left(p_{2}-1\right)}} \beta^{\frac{2}{3\left(p_{2}-1\right)}}+(1-\alpha)^{\frac{7 p_{1}-9}{3\left(p_{1}-1\right)}}(1-\beta)^{\frac{2}{3\left(p_{1}-1\right)}} \leq \alpha^{\frac{7 p_{1}-9}{3\left(p_{1}-1\right)}}+(1-\alpha)^{\frac{7 p_{1}-9}{3\left(p_{1}-1\right)}}<1,
$$

where we used (4.10), and (1.24) follows. This concludes the proof of Lemma 1.5.

Remark 4.2 From the above proof, (1.24) is satisfied as long as $j$ satisfies (1.26) with $\frac{9}{7}<p_{1} \leq p_{2}<+\infty$ such that for all $0<\alpha<1,0 \leq \beta \leq 1$,

$$
\alpha^{\frac{7 p_{2}-9}{3\left(p_{2}-1\right)}} \beta^{\frac{2}{3\left(p_{2}-1\right)}}+(1-\alpha)^{\frac{7 p_{1}-9}{3\left(p_{1}-1\right)}}(1-\beta)^{\frac{2}{3\left(p_{1}-1\right)}}<1 .
$$

The condition $p_{1}>\frac{3}{2}$ is just one among the very many possibilities. Now observe on the other hand that the property does not hold true in the limiting case $p_{1}=\frac{9}{7}, p_{2}=+\infty$.

\subsection{Compactness of the minimizing sequence for $N=3$}

This subsection is devoted to the proof of Theorem 1.4, which states that the sequences $f_{n}$ minimizing the energy under the constraints $\left|f_{n}\right|_{L^{1}}$ and $\left|j\left(f_{n}\right)\right|_{L^{1}}$ fixed are up to a translation shift relatively compact in $\mathcal{E}_{p}$. Recall that we work in dimension $N=3$. We consider simultaneously the minimization problems (1.23) and (1.25).

\section{Proof of Theorem 1.4}

Let $f_{n}$ be a minimizing sequence for (1.23):

$$
\left|f_{n}\right|_{L^{1}}=M_{1}, \quad\left|j\left(f_{n}\right)\right|_{L^{1}}=M_{j}, \quad \mathcal{H}\left(f_{n}\right) \rightarrow I\left(M_{1}, M_{j}\right) \text { as } n \rightarrow+\infty,
$$

or for the minimization problem (1.25):

$$
\left|f_{n}\right|_{L^{1}}=M_{1}, \quad\left|f_{n}\right|_{L^{\infty}}=M_{\infty}, \quad \mathcal{H}\left(f_{n}\right) \rightarrow I\left(M_{1}, M_{\infty}\right) \quad \text { as } n \rightarrow+\infty .
$$

First observe from interpolation estimate (1.5) that

$$
\limsup _{n \rightarrow+\infty}\left|f_{n}\right| \mathcal{E}_{p}<+\infty
$$

where either $p_{\text {crit }}<p<+\infty$ is given from (1.22) or $p=+\infty$. This enables us to apply the concentration compactness Lemma 3.1 and we claim from the estimates of Lemma 3.2 that only compactness may occur.

Step 1 Vanishing cannot occur.

Observe that

$$
\left|E_{f_{n}}\right|_{L^{2}}^{2}=-\mathcal{H}\left(f_{n}\right)+\int_{\mathbb{R}^{2 N}}|v|^{2} f_{n}(x, v) d x d x
$$


Therefore (4.13) implies the uniform lower bound on the potential energy:

$$
\liminf _{n \rightarrow+\infty}\left|E_{f_{n}}\right|_{L^{2}}^{2} \geq-I\left(M_{1}, M_{p}\right)>0
$$

by (4.1). Remark that (4.1) holds as well with the same proof for (1.25). If vanishing occurs, (3.9) contradicts (4.15).

Step 2 Dichotomy cannot occur.

Assume now that dichotomy occurs. Pick an $\varepsilon>0$. From (3.4), we may assume without loss of generality that

$$
\lim _{k \rightarrow+\infty}\left|f_{k}^{1}\right|_{L^{1}}=\alpha M_{1}, \quad \lim _{k \rightarrow+\infty}\left|f_{k}^{2}\right|_{L^{1}}=(1-\alpha) M_{1}
$$

for some

$$
0<\alpha<1 \text { independent of } \varepsilon \text {. }
$$

Consider first the case of the minimization problem (1.23). Thanks to the disjoint support properties of $f_{k}^{1}, f_{k}^{2}, v_{k}$ given by (3.4), and thanks to (4.13), we may also assume that

$$
\lim _{k \rightarrow+\infty}\left|j\left(f_{k}^{1}\right)\right|_{L^{1}}=M_{j}^{1}, \quad \lim _{k \rightarrow+\infty}\left|j\left(f_{k}^{2}\right)\right|_{L^{1}}=M_{j}^{2}
$$

with

$$
M_{j}^{1}+M_{j}^{2} \leq M_{j}
$$

Notice that (3.4) also implies

$$
\int_{\mathbb{R}^{2 N}}|v|^{2} f_{n_{k}}(x, v) d x d v \geq \int_{\mathbb{R}^{2 N}}|v|^{2} f_{k}^{1}(x, v) d x d v+\int_{\mathbb{R}^{2 N}}|v|^{2} f_{k}^{2}(x, v) d x d v .
$$

Next, we plug (3.10) and (4.19) into (4.13) to derive

$$
I\left(M_{1}, M_{j}\right) \geq \limsup _{k \rightarrow+\infty} \mathcal{H}\left(f_{k}^{1}\right)+\limsup _{k \rightarrow+\infty} \mathcal{H}\left(f_{k}^{2}\right)-C \varepsilon^{\theta} .
$$

Letting $\varepsilon \rightarrow 0$, we thus have the existence of $0<\alpha<1$ and $M_{j}^{1}, M_{j}^{2}$ satisfying (4.18) and

$$
I\left(M_{1}, M_{j}\right) \geq I\left(\alpha M_{1}, M_{j}^{1}\right)+I\left((1-\alpha) M_{1}, M_{j}^{2}\right) .
$$

We claim that this contradicts (1.24). Indeed, from (4.18) we may write

$$
M_{j}^{1}=\beta\left(M_{j}^{1}+M_{j}^{2}\right) \leq \beta M_{j}, \quad M_{j}^{2}=(1-\beta)\left(M_{j}^{1}+M_{j}^{2}\right) \leq(1-\beta) M_{j}
$$

for some $0 \leq \beta \leq 1$. Thanks to (4.2), one deduces from (4.20) that

$$
I\left(M_{1}, M_{j}\right) \geq I\left(\alpha M_{1}, \beta M_{j}\right)+I\left((1-\alpha) M_{1},(1-\beta) M_{j}\right),
$$


which contradicts (1.24).

Consider now the case of minimization problem (1.25). Direct computations lead to

$$
\forall 0 \leq \alpha, \beta \leq 1, \quad I\left(\alpha M_{1}, \beta M_{\infty}\right)=\alpha^{\frac{7}{3}} \beta^{\frac{2}{3}} I\left(M_{1}, M_{\infty}\right) \geq \alpha^{\frac{7}{3}} I\left(M_{1}, M_{\infty}\right)
$$

and we thus obtain from (4.20):

$$
I\left(M_{1}, M_{\infty}\right) \geq\left(\alpha^{\frac{7}{3}}+(1-\alpha)^{\frac{7}{3}}\right) I\left(M_{1}, M_{\infty}\right)
$$

with $0<\alpha<1$. A contradiction now follows from (4.10).

Step 3 Conclusion.

We conclude from Lemma 3.1 that compactness occurs. Consider first the minimization problem (1.23). From Lemma 3.2, up to a subsequence, there exists a shift $y_{n} \in \mathbb{R}^{N}$ such that (3.6) occurs. Let $\bar{f}_{n}(x, v)=f_{n}\left(x+y_{n}, v\right)$, then from Fatou's lemma,

$$
\liminf _{n \rightarrow+\infty} \int_{\mathbb{R}^{2 N}}|v|^{2} \bar{f}_{n}(x, v) d x d v \geq \int_{\mathbb{R}^{2 N}}|v|^{2} f(x, v) d x d v .
$$

By lower semicontinuity, we also have

$$
\liminf _{n \rightarrow+\infty}\left|j\left(f_{n}\right)\right|_{L^{1}} \geq|j(f)|_{L^{1}}
$$

We thus conclude from (3.8), (4.13) that $f$ satisfies

$$
|f|_{L^{1}}=M_{1}, \quad M_{j} \geq|j(f)|_{L^{1}}, \quad I\left(M_{1}, M_{j}\right) \geq \mathcal{H}(f) .
$$

We claim that

$$
|j(f)|_{L^{1}}=M_{j} .
$$

Indeed, let $\alpha=\frac{|j(f)|_{L^{1}}}{M_{j}}$. If $\alpha<1$, then arguing as for the proof of (4.2) ie considering a suitable rescaled version of $f$, we easily derived from (4.22):

$$
I\left(|f|_{L^{1}},|j(f)|_{L^{1}}\right)=I\left(M_{1}, \alpha M_{j}\right)>I\left(M_{1}, M_{j}\right) \geq \mathcal{H}(f),
$$

which contradicts the definition of $I\left(|f|_{L^{1}},|j(f)|_{L^{1}}\right)$. Therefore (4.23) holds, and together with (4.22), this implies that $f$ is a minimizer:

$$
|f|_{L^{1}}=M_{1}, \quad|j(f)|_{L^{1}}=M_{j}, \quad \mathcal{H}(f)=I\left(M_{1}, M_{j}\right) .
$$

In particular,

$$
\left|\bar{f}_{n}\right|_{L^{1}} \rightarrow|f|_{L^{1}}, \quad\left|j\left(\bar{f}_{n}\right)\right|_{L^{1}} \rightarrow|j(f)|_{L^{1}},\left.\left.\left.\left.\quad|| v\right|^{2} \bar{f}_{n}\right|_{L^{1}} \rightarrow|| v\right|^{2} f\right|_{L^{1}}
$$

We now claim that this implies

$$
\bar{f}_{n} \rightarrow f \text { in } \mathcal{E}_{p}
$$


To prove (4.25), we adapt an argument from [24], section II. Let

$$
g_{n}=\frac{\bar{f}_{n}+f}{2} .
$$

By convexity, we have $j\left(g_{n}\right) \leq \frac{1}{2}\left(j\left(\bar{f}_{n}\right)+j(f)\right)$, and (3.8) gives $E_{g_{n}}=\frac{1}{2}\left(E_{\bar{f}_{n}}+E_{f}\right) \rightarrow E_{f}$ in $L^{2}$. We thus have

$$
\left|g_{n}\right|_{L^{1}}=M_{1}, \quad \mathcal{H}\left(g_{n}\right) \rightarrow I\left(M_{1}, M_{j}\right), \quad \limsup _{n \rightarrow+\infty}\left|j\left(g_{n}\right)\right|_{L^{1}} \leq M_{j}
$$

where we also used (4.24). Arguing as for the proof of (4.23), we conclude that $\left|j\left(g_{n}\right)\right|_{L^{1}} \rightarrow$ $M_{j}=|j(f)|_{L^{1}}$ as $n \rightarrow+\infty$, from which

$$
j\left(\bar{f}_{n}\right)+j(f)-2 j\left(g_{n}\right) \rightarrow 0 \text { in } L^{1} \text { as } n \rightarrow+\infty .
$$

For given parameters $K, \eta>0$, define

$$
\delta_{K}(\eta)=\inf \left\{j(s)+j(t)-2 j\left(\frac{s+t}{2}\right), \quad 0 \leq s \leq K, \quad 0 \leq t \leq K, \quad|s-t| \geq \eta\right\} .
$$

The strict convexity of $j$ ensures that $\delta_{K}(\eta)>0$. Let

$$
\Omega_{K, \eta}=\left\{X \in \mathbb{R}^{6}: \quad \bar{f}_{n}(X) \leq K, \quad f(X) \leq K, \quad\left|\bar{f}_{n}(X)-f(X)\right| \geq \eta\right\} .
$$

Remarking that

$$
\operatorname{meas}\left\{X \in \mathbb{R}^{6}: \quad \bar{f}_{n}(X) \geq K \text { or } f(X) \geq K\right\} \leq \frac{2 M_{1}}{K}
$$

and that $\delta_{K}(\eta)$ is a non decreasing function of $\eta$, we have for all $\eta, K>0$,

$$
\begin{aligned}
\operatorname{meas}\{X & \left.\in \mathbb{R}^{6}:\left|\bar{f}_{n}(X)-f(X)\right| \geq \eta\right\} \\
& \leq \frac{2 M_{1}}{K}+\operatorname{meas}\left\{X \in \Omega_{K, \eta}: \quad \delta_{K}\left(\left|\bar{f}_{n}(X)-f(X)\right|\right) \geq \delta_{K}(\eta)\right\} \\
& \leq \frac{2 M_{1}}{K}+\frac{1}{\delta_{K}(\eta)} \int_{\Omega_{K, \eta}} \delta_{K}\left(\left|\bar{f}_{n}(X)-f(X)\right|\right) d X \\
& \leq \frac{2 M_{1}}{K}+\frac{1}{\delta_{K}(\eta)} \int_{\mathbb{R}^{6}}\left(j\left(\bar{f}_{n}(X)\right)+j(f(X))-j\left(g_{n}(X)\right) d X .\right.
\end{aligned}
$$

We thus conclude that

$$
\forall \eta>0, \quad \operatorname{meas}\left\{X \in \mathbb{R}^{6}, \quad\left|\bar{f}_{n}(X)-f(X)\right| \geq \eta\right\} \rightarrow 0 \text { as } n \rightarrow+\infty .
$$

By (4.24), this implies

$$
\bar{f}_{n} \rightarrow f, \quad|v|^{2} \bar{f}_{n} \rightarrow|v|^{2} f \quad j\left(\bar{f}_{n}\right) \rightarrow j(f) \quad \text { in } L^{1} .
$$


Moreover, from (1.22), there holds

$$
\forall \eta>0, \quad \exists R(\eta) \text { such that } \forall n \geq 1, \quad \int_{|X| \geq R(\eta)}\left|\bar{f}_{n}\right|^{p} \leq C \int_{|X| \geq R(\eta)} j\left(\bar{f}_{n}\right)<\eta
$$

and thus (4.27) implies that $\bar{f}_{n} \rightarrow f$ in $L^{p}$. This concludes the proof of (4.25).

Consider now the minimization problem (1.25). From Lemma 3.2, (3.7) occurs with

$$
|f|_{L^{1}}=M_{1}, \quad M_{\infty} \geq|f|_{L^{\infty}}, \quad I\left(M_{1}, M_{\infty}\right) \geq \mathcal{H}(f) .
$$

By (4.21), it is clear that $|f|_{L^{\infty}}=M_{\infty}$ and the conclusion follows from Theorem 1.3. This concludes the proof of Theorem 1.4.

\subsection{Orbital stability of the ground states for $N=3$}

Let us now prove Corollary 1.6. Arguing by contradiction and using (1.3) and (1.4), we equivalently need to prove the following:

Proposition 4.3 Let $\frac{9}{7}<p \leq \infty$ and $Q_{p}$ be the ground state. Let $\left(f_{n}\right)_{n \geq 1}$ a sequence in $L_{+}^{1}\left(\mathbb{R}^{2 N}\right)$ such that:

$$
\limsup _{n \rightarrow+\infty}\left|f_{n}\right|_{L^{1}} \leq\left|Q_{p}\right|_{L^{1}}, \quad \limsup _{n \rightarrow+\infty}\left|f_{n}\right|_{L^{p}} \leq\left|Q_{p}\right|_{L^{p}}, \quad \limsup _{n \rightarrow+\infty} \mathcal{H}\left(f_{n}\right) \leq \mathcal{H}\left(Q_{p}\right),
$$

then up to a subsequence, there exists a translation shift $y_{n} \in \mathbb{R}^{N}$ such that

$$
\tilde{f}_{n}(x, v)=f_{n}\left(x+y_{n}, v\right)
$$

satisfies

$$
\tilde{f}_{n} \rightarrow Q_{p} \text { in } \mathcal{E}_{p} \text { in the case } p<+\infty
$$

or

$$
\tilde{f}_{n} \rightarrow Q_{\infty} \text { in } L^{1} \text { and } L^{\infty} \text { weak } \star \text { in the case } p=+\infty .
$$

\section{Proof of Proposition 4.3}

We claim that $f_{n}$ is a minimizing sequence for (1.23) ie

$$
\left|f_{n}\right|_{L^{1}} \rightarrow\left|Q_{p}\right|_{L^{1}}, \quad\left|f_{n}\right|_{L^{p}} \rightarrow\left|Q_{p}\right|_{L^{p}}, \quad \mathcal{H}\left(f_{n}\right) \rightarrow \mathcal{H}\left(Q_{p}\right),
$$

and conclusion thus follows from Theorem 1.4 with $j(t)=t^{p}$, completed with Lemma 1.5. To prove (4.29), we simply remark from (4.8) that

$$
\mathcal{H}\left(f_{n}\right) \geq I\left(\left|f_{n}\right|_{L^{1}},\left|f_{n}\right|_{L^{p}}^{p}\right)=\left(\frac{\left|f_{n}\right|_{L^{1}}}{\left|Q_{p}\right|_{L^{1}}}\right)^{\frac{7 p-9}{3(p-1)}}\left(\frac{\left|f_{n}\right|_{L^{p}}}{\left|Q_{p}\right|_{L^{p}}}\right)^{\frac{2 p}{3(p-1)}} \mathcal{H}\left(Q_{p}\right)
$$

and conclusion follows from $(4.28)$ and $\mathcal{H}\left(Q_{p}\right)<0$. This concludes the proof of Proposition 4.3 for $p<\infty$.

The case $p=+\infty$ can be treated with the same strategy, by simply using (4.21) instead of (4.8) in order to show that $f_{n}$ is a minimizing sequence for (1.25). 


\section{Proof of the stability result for $N=4$}

This section is devoted to the proofs of the blow up results in dimension $N=4$. We first prove the orbital stability of the polytrope $Q_{p}$ as a blow up profile i.e. Proposition 1.11. We then prove in subsection 5.2 the mass concentration phenomenon for finite time blow up solutions as claimed by Theorem 1.12 .

\subsection{Orbital stability of the ground states for $N=4$}

This subsection is devoted to the proof of Proposition 1.11. Arguing by contradiction, we equivalently need to prove the following:

Proposition 5.1 Let $2<p \leq \infty$ and $Q_{p}$ be the ground state. Let $\left(f_{n}\right)_{n \geq 1}$ a sequence in $L_{+}^{1}\left(\mathbb{R}^{2 N}\right)$ such that:

$$
\limsup _{n \rightarrow+\infty}\left|f_{n}\right|_{L^{1}} \leq\left|Q_{p}\right|_{L^{1}}, \quad \limsup _{n \rightarrow+\infty}\left|f_{n}\right|_{L^{p}} \leq\left|Q_{p}\right|_{L^{p}}, \quad \limsup _{n \rightarrow+\infty} \frac{\mathcal{H}\left(f_{n}\right)}{\left.\left.|| v\right|^{2} f_{n}\right|_{L^{1}}} \leq 0 .
$$

Let

$$
\lambda_{n}=\left(\frac{\left.\left.|| v\right|^{2} Q_{p}\right|_{L^{1}}}{\left.\left.|| v\right|^{2} f_{n}\right|_{L^{1}}}\right)^{\frac{1}{2}}
$$

then up to a subsequence, there exists a translation shift $y_{n} \in \mathbb{R}^{N}$ such that

$$
\tilde{f}_{n}(x, v)=f_{n}\left(\lambda_{n}\left(x+y_{n}\right), \frac{v}{\lambda_{n}}\right)
$$

satisfies

$$
\begin{gathered}
\tilde{f}_{n} \rightarrow Q_{p} \text { in } \mathcal{E}_{p} \text { for } p<+\infty, \\
\tilde{f}_{n} \rightarrow Q_{\infty} \text { in } L^{1} \text { and } L^{\infty} \text { weak } \star \text { for } p=+\infty .
\end{gathered}
$$

\section{Proof of Proposition 5.1}

Let $\hat{f}_{n}(x, v)=f_{n}\left(\lambda_{n} x, \frac{v}{\lambda_{n}}\right)$ with $\lambda_{n}$ given by (5.2). Then

$$
\left|\hat{f}_{n}\right|_{L^{1}}=\left|f_{n}\right|_{L^{1}}, \quad\left|\hat{f}_{n}\right|_{L^{p}}=\left|f_{n}\right|_{L^{p}},\left.\left.\quad|| v\right|^{2} \hat{f}_{n}\right|_{L^{1}}=\left.\left.|| v\right|^{2} Q_{p}\right|_{L^{1}}, \quad \frac{\mathcal{H}\left(\hat{f}_{n}\right)}{\left.\left.|| v\right|^{2} \hat{f}_{n}\right|_{L^{1}}}=\frac{\mathcal{H}\left(f_{n}\right)}{\left.\left.|| v\right|^{2} f_{n}\right|_{L^{1}}}
$$

and we have from (5.1)

$$
\limsup _{n \rightarrow+\infty}\left|\hat{f}_{n}\right|_{L^{1}} \leq\left|Q_{p}\right|_{L^{1}}, \quad \limsup _{n \rightarrow+\infty}\left|\hat{f}_{n}\right|_{L^{p}} \leq\left|Q_{p}\right|_{L^{p}}, \quad \limsup _{n \rightarrow+\infty} \mathcal{H}\left(\hat{f}_{n}\right) \leq 0 .
$$

We claim

$$
\left|\hat{f}_{n}\right|_{L^{1}} \rightarrow\left|Q_{p}\right|_{L^{1}}, \quad\left|\hat{f}_{n}\right|_{L^{p}} \rightarrow\left|Q_{p}\right|_{L^{p}},\left.\left.\quad|| v\right|^{2} \hat{f}_{n}\right|_{L^{1}}=\left.\left.|| v\right|^{2} Q_{p}\right|_{L^{1}}, \quad \limsup _{n \rightarrow+\infty} \mathcal{H}\left(\hat{f}_{n}\right) \leq 0 .
$$


Indeed, from (1.28),

$$
\begin{aligned}
\mathcal{H}\left(\hat{f}_{n}\right) \geq & \geq\left.|v|^{2} \hat{f}_{n}\right|_{L^{1}}\left(1-\left[\frac{\left|\hat{f}_{n}\right|_{L^{1}}\left|\hat{f}_{n}\right|_{L^{p}}^{\frac{p}{p-2}}}{\left|Q_{p}\right|_{L^{1}}\left|Q_{p}\right|_{L^{p}}^{\frac{p}{p-2}}}\right]^{\frac{p-2}{2(p-1)}}\right) \\
& =\left.\left.|| v\right|^{2} Q_{p}\right|_{L^{1}}\left(1-\left[\frac{\left|\hat{f}_{n}\right|_{L^{1}}\left|\hat{f}_{n}\right|_{L^{p}}^{\frac{p}{p-2}}}{\left|Q_{p}\right|_{L^{1}}\left|Q_{p}\right|_{L^{p}}^{\frac{p}{p-2}}}\right]^{\frac{p-2}{2(p-1)}}\right)
\end{aligned}
$$

and the conclusion follows from (5.5).

From (5.6), we may apply concentration compactness Lemmas 3.1 and 3.2 to $\hat{f}_{n}$ and claim again that up to a subsequence, only compactness may occur. To this aim, observe first that

$$
\mathcal{H}\left(\hat{f}_{n}\right)=\int_{\mathbb{R}^{2 N}}|v|^{2} \hat{f}_{n}(x, v) d x d x-\left|E_{\hat{f}_{n}}\right|_{L^{2}}^{2}=\left.\left.|| v\right|^{2} Q_{p}\right|_{L^{1}}-\left|E_{\hat{f}_{n}}\right|_{L^{2}}^{2}
$$

and thus (5.6) implies the uniform lower bound on the potential energy

$$
\liminf _{n \rightarrow+\infty}\left|E_{\hat{f}_{n}}\right|_{L^{2}}^{2} \geq\left.\left.|| v\right|^{2} Q_{p}\right|_{L^{1}}>0
$$

Step 1 Vanishing cannot occur.

If vanishing occurs, then (5.7) contradicts (3.9).

Step 2 Dichotomy cannot occur.

Assume now that dichotomy occurs. Pick an $\varepsilon>0$ and observe from (3.4) that

$$
\int_{\mathbb{R}^{2 N}}|v|^{2} \hat{f}_{n_{k}}(x, v) d x d v \geq \int_{\mathbb{R}^{2 N}}|v|^{2} \hat{f}_{k}^{1}(x, v) d x d v+\int_{\mathbb{R}^{2 N}}|v|^{2} \hat{f}_{k}^{2}(x, v) d x d v
$$

and thus from (5.6) and (3.10):

$$
0 \geq \limsup _{k \rightarrow+\infty} \mathcal{H}\left(f_{k}^{1}\right)+\limsup _{k \rightarrow+\infty} \mathcal{H}\left(f_{k}^{2}\right)-C \varepsilon^{\theta},
$$

with $\theta>0$. Moreover, from (3.4),

$$
\int_{\mathbb{R}^{2 N}}\left(\hat{f}_{n_{k}}\right)^{p} \geq \int_{\mathbb{R}^{2 N}}\left(\hat{f}_{k}^{i}\right)^{p}, \quad i=1,2
$$

and there exists $0<\alpha<1$ such that

$$
\left.\limsup _{k \rightarrow+\infty}|| f_{k}^{1}\right|_{L^{1}}-\alpha\left|Q_{p}\right|_{L^{1}}\left|+\limsup _{k \rightarrow+\infty}\right|\left|f_{k}^{2}\right|_{L^{1}}-(1-\alpha)\left|Q_{p}\right|_{L^{1}} \mid<\varepsilon .
$$


This implies

$$
\liminf _{k \rightarrow \infty}\left(1-\left[\frac{\left|\hat{f}_{k}^{i}\right|_{L^{1}}\left|\hat{f}_{k}^{i}\right|_{L^{p}}^{\frac{p}{p-2}}}{\left|Q_{p}\right|_{L^{1}}\left|Q_{p}\right|_{L^{p}}^{\frac{p}{p-2}}}\right]^{\frac{p-2}{2(p-1)}}\right) \geq C>0, \quad i=1,2
$$

for $\varepsilon>0$ small enough. We then conclude using (5.8) and sharp interpolation estimate (1.28) that

$$
\left.\left.\limsup _{k \rightarrow+\infty}|| v\right|^{2} \hat{f}_{k}^{i}\right|_{L^{1}} \leq C \varepsilon^{\theta} \quad i=1,2
$$

from which

$$
\limsup _{k \rightarrow+\infty}\left|E_{\hat{f}_{k}^{i}}\right|_{L^{2}} \leq C \varepsilon^{\theta}, \quad i=1,2 .
$$

From (3.10), we conclude

$$
\limsup _{k \rightarrow+\infty}\left|E_{\hat{f}_{n_{k}}}\right|_{L^{2}} \leq C \varepsilon^{\theta}
$$

which contradicts (5.7) for $\varepsilon>0$ small enough.

\section{Step 3 Conclusion}

We conclude that only compactness occurs. Thus from Lemma 3.2, there exists a shift $y_{n} \in \mathbb{R}^{N}$ such that up to a subsequence, $\tilde{f}_{n}(x, v)=\hat{f}_{n}\left(x+y_{n}, v\right)$ satisfies (3.6) or (3.7). From Fatou's lemma and lower semicontinuity, we estimate

$$
\liminf _{n \rightarrow+\infty} \int_{\mathbb{R}^{2 N}}|v|^{2} \tilde{f}_{n}(x, v) d x d v \geq \int_{\mathbb{R}^{2 N}}|v|^{2} f(x, v) d x d v, \quad \liminf _{n \rightarrow+\infty}\left|\tilde{f}_{n}\right|_{L^{p}} \geq|f|_{L^{p}},
$$

and thus from (3.8) and (5.6),

$$
|f|_{L^{1}}=\left|Q_{p}\right|_{L^{1}}, \quad\left|Q_{p}\right|_{L^{p}} \geq|f|_{L^{p}}, \quad \mathcal{H}(f) \leq 0 .
$$

From the variational characterization (1.18) of $Q_{p}$, there exists $x_{0} \in \mathbb{R}^{4}$ such that

$$
f=Q_{p}\left(\cdot-x_{0}\right)
$$

This concludes the proof of (5.4) for $p=+\infty$. In the case $p<+\infty$, the strong $L^{p}$ convergence (5.3) follows from $\left|\tilde{f}_{n}\right|_{L^{p}} \rightarrow|Q|_{L^{p}}=|f|_{L^{p}}$ as $n \rightarrow+\infty$, and, in a second step, the strong convergence in $\mathcal{E}_{p}$ follows from $\left|\tilde{f}_{n}\right|_{L^{1}} \rightarrow|f|_{L^{1}}$ and $\left.\left.\left.\left.|| v\right|^{2} \tilde{f}_{n}\right|_{L^{1}} \rightarrow|| v\right|^{2} f\right|_{L^{1}}$. This concludes the proof of Proposition 5.1. 


\subsection{Proof of the mass concentration phenomenon}

This subsection is devoted to the proof of Theorem 1.12. The heart of the proof is the following compactness Lemma.

Lemma 5.2 Let $2<p<+\infty, M_{1}, M_{p}>0$ and a sequence $f_{n} \in \mathcal{E}_{p}$ with

$$
\left|f_{n}\right|_{L^{1}} \leq M_{1}, \quad\left|f_{n}\right|_{L^{p}} \leq M_{p},\left.\left.\quad|| v\right|^{2} f_{n}\right|_{L^{1}}=1, \quad \limsup _{n \rightarrow+\infty} \mathcal{H}\left(f_{n}\right) \leq 0 .
$$

Then there exists $\beta>0$ depending only on $M_{1}$ and $M_{p}$ and a translation shift $y_{n} \in \mathbb{R}^{N}$ such that up to a subsequence,

$$
f_{n}\left(x+y_{n}, v\right) \rightarrow f \quad \text { in } L^{p}
$$

with

$$
f \in \mathcal{E}_{p}, \quad|f|_{L^{1}} \geq \beta>0 \quad \text { and } \quad \mathcal{H}(f) \leq 0 .
$$

Let us first assume Lemma 5.2 and conclude the proof of Theorem 1.12.

\section{Proof of Theorem 1.12}

We argue by contradiction and assume that there exists $\eta>0,0<R<+\infty$ and a sequence $t_{n} \rightarrow T$ such that $\forall n \geq 1$,

$$
\sup _{y \in \mathbb{R}^{N}}\left(\int_{y+B_{R}} \rho\left(t_{n}, x\right) d x\right)\left(\int_{y+B_{R}} \rho_{p}^{p}\left(t_{n}, x\right) d x\right)^{\frac{1}{p-2}}<\left|Q_{p}\right|_{L^{1}}\left|Q_{p}\right|_{L^{p}}^{\frac{p}{p-2}}-\eta .
$$

Let

$$
\tilde{f}_{n}(x, v)=f_{n}\left(t_{n}, \lambda_{n} x, \frac{v}{\lambda_{n}}\right) \quad \text { with } \quad \lambda_{n}=\left(\frac{1}{\left.\left.|| v\right|^{2} f\left(t_{n}\right)\right|_{L^{1}}}\right)^{\frac{1}{2}}
$$

then

$$
\left.\left.|| v\right|^{2} \tilde{f}_{n}\right|_{L^{1}}=1 \quad \text { and } \quad \limsup _{n \rightarrow+\infty} \mathcal{H}\left(\tilde{f}_{n}\right)=\limsup _{n \rightarrow+\infty}\left(\lambda_{n}^{2} \mathcal{H}\left(f_{n}\right)\right) \leq 0
$$

from (1.31) and (1.3). Next, from the control of the $L^{1}$ and the $L^{p}$ norm (1.4), $\tilde{f}_{n}$ satisfies the assumptions of Lemma 5.2. Consequently, there exists $y_{n} \in \mathbb{R}^{N}$ such that, up to a subsequence, $\bar{f}_{n}(x, v)=\tilde{f}_{n}\left(x+y_{n}, v\right) \rightarrow f$ in $L^{p}$. Moreover, from (5.10), $f$ is non zero and $\mathcal{H}(f) \leq 0$, thus from $(1.28)$,

$$
|f|_{L^{1}}|f|_{L^{p}}^{\frac{p}{p-2}} \geq\left|Q_{p}\right|_{L^{1}}\left|Q_{p}\right|_{L^{p}}^{\frac{p}{p-2}} .
$$

Now from Fatou's Lemma and $\lambda_{n} \rightarrow 0$ as $n \rightarrow+\infty$, we have: $\forall A>0$,

$$
\begin{aligned}
& \iint_{|v|+|y| \leq A} f(x, v) d x d v \leq \liminf _{n \rightarrow+\infty} \iint_{|v|+|y| \leq A} \bar{f}_{n}(x, v) d x d v \\
= & \liminf _{n \rightarrow+\infty} \iint_{|v|+\left|y-y_{n}\right| \leq A} \tilde{f}_{n}(x, v) d x d v \leq \liminf _{n \rightarrow+\infty} \int_{\left|y-y_{n}\right| \leq \frac{R}{\lambda_{n}}} \rho_{\tilde{f}_{n}}(x) d x \\
= & \liminf _{n \rightarrow+\infty} \int_{\left|y-y_{n}\right| \leq R} \rho\left(t_{n}, x\right) d x
\end{aligned}
$$


and similarly for the $L^{p}$ norm. We conclude from (5.11) and letting $A \rightarrow+\infty$ that

$$
|f|_{L^{1}}|f|_{L^{p}}^{\frac{p}{p-2}}<\left|Q_{p}\right|_{L^{1}}\left|Q_{p}\right|_{L^{p}}^{\frac{p}{p-2}}-\eta
$$

which contradicts (5.12) and concludes the proof of Theorem 1.12.

\section{Proof of Lemma 5.2}

We borrow a very elegant inductive argument from [29]. Let us first define the positive constant $\beta$. We observe that (5.9) implies the uniform lower bound on the potential energy

$$
\liminf _{n \rightarrow+\infty}\left|E_{f_{n}}\right|_{L^{2}}^{2} \geq 1
$$

Then a close look at the proof of (3.9) shows that this lower bound together with the $L^{1}$ and $L^{p}$ bounds on $f_{n}$ enables us to find $\beta_{1}>0$ and $R>0$, depending only on $M_{1}$ and $M_{p}$, such that

$$
\liminf _{n \rightarrow+\infty} \sup _{y \in \mathbb{R}^{4}} \int_{y+B_{R}} \rho_{n}(x) d x \geq \beta_{1}
$$

Hence by choosing

$$
\beta=\min \left(\beta_{1}, \frac{\left|Q_{p}\right|_{L^{1}}\left|Q_{p}\right|_{L^{p}}^{\frac{p}{p-2}}}{M_{p}}\right),
$$

we ensure, first, that for any sequence $f_{n} \in \mathcal{E}_{p}$ satisfying (5.9) there exists a shift $y_{n} \in \mathbb{R}^{4}$ such that

$$
\liminf _{n \rightarrow+\infty} \int_{\left|y-y_{n}\right|<R} \rho_{f_{n}}(x) d x \geq \beta
$$

and, second, from (1.28), that any function $g$ satisfying $|g|_{L^{1}} \leq \beta,|g|_{L^{p}} \leq M_{p}$ has a nonnegative energy.

Let $f_{n}$ satisfy (5.9). Since

$$
\limsup _{n \rightarrow+\infty} \mathcal{H}\left(f_{n}\right) \leq 0,\left.\left.\quad|| v\right|^{2} f_{n}\right|_{L^{1}}=1
$$

from the sharp interpolation estimate (1.28), we deduce that

$$
\liminf _{n \rightarrow+\infty}\left|f_{n}\right|_{L^{1}}\left|f_{n}\right|_{L^{p}}^{\frac{p}{p-2}} \geq\left|Q_{p}\right|_{L^{1}}\left|Q_{p}\right|_{L^{p}}^{\frac{p}{p-2}}
$$

Thanks to the upper bounds in (5.9) and up to a subsequence, one has

$$
\left|f_{n}\right|_{L^{1}} \rightarrow \tilde{M}_{1}>0 \quad \text { and } \quad\left|f_{n}\right|_{L^{p}} \rightarrow \tilde{M}_{p}>0 \quad \text { as } n \rightarrow+\infty .
$$

Let $D$ be the unique integer such that

$$
D \beta \leq \tilde{M}_{1}<(D+1) \beta .
$$


From (5.14) it is clear that $D \geq 1$. In order to prove the Lemma, we shall proceed by induction on $D$.

Assume first that $D=1$ and observe that $f_{n}$ satisfies the assumptions of Lemma 3.1. By (5.13), vanishing cannot occur. If dichotomy occurs, then by diagonal extraction on the sequences $f_{k}^{1}, f_{k}^{2}$ of Lemma 3.1 and from explicit formulas (E.7), we may assume without loss of generality the following: there exist $m \in\left(0, \tilde{M}_{1}\right), \tilde{y}_{k} \in \mathbb{R}^{4}, f_{k}^{1}, f_{k}^{2}$, such that as $k \rightarrow+\infty$

$$
\begin{gathered}
f_{k}^{1} \rightarrow f^{1} \quad \text { and } \quad f_{n_{k}}\left(x+\tilde{y}_{k}, v\right) \rightarrow f^{1} \text { in } L^{p}, \quad E_{f_{k}^{1}} \rightarrow E_{f^{1}} \quad \text { in } L^{2}, \\
\left|f_{n_{k}}\right|_{L^{1}} \rightarrow\left|f^{1}\right|_{L^{1}}=m, \quad\left|f_{k}^{2}\right|_{L^{1}} \rightarrow \tilde{M}_{1}-m,\left.\left.\quad|| v\right|^{2} f_{k}^{2}\right|_{L^{1}} \leq 1, \quad\left|f_{k}^{2}\right|_{L^{p}} \leq M_{p}, \\
\left|E_{f_{n_{k}}}\right|_{L^{2}}^{2}-\left|E_{f_{k}^{2}}\right|_{L^{2}}^{2}-\left|E_{f^{1}}\right|_{L^{2}}^{2} \rightarrow 0 \quad \text { as } k \rightarrow+\infty,
\end{gathered}
$$

where we used (3.10). Observe from (5.14) and the definition of $m$ (E.1) that $\left|f^{1}\right|_{L^{1}}=$ $m \geq \beta$. From the assumption $D=1$, this implies $\left|f_{k}^{2}\right|_{L^{1}}<\beta$ for $k$ large enough, from which

$$
\mathcal{H}\left(f_{k}^{2}\right) \geq 0
$$

for $k$ large enough. We conclude that

$$
\mathcal{H}\left(f^{1}\right) \leq 0
$$

as wanted. If compactness occurs then we have directly $\bar{f}_{n}(x, v)=f_{n}\left(x+\tilde{y}_{n}, v\right) \rightarrow f$ in $L^{p}$ with, from (3.8) and (5.13), $\left|E_{f}\right|_{L^{2}} \geq 1$. This is enough to conclude $\mathcal{H}(f) \leq 0$. Moreover by (3.8) we also have $|f|_{L^{1}}=M_{1} \geq \beta$, as wanted.

We now assume the result proved for $D_{0}$ and prove it for $D_{0}+1$. Again, vanishing cannot occur since $f_{n}$ satisfies (5.14). If compactness occurs, then the claim follows as before. If dichotomy occurs, we argue as above and extract $f_{k}^{1} \rightarrow f^{1}$ in $L^{p}$ with $\left|f^{1}\right|_{L^{1}} \geq \beta$, from which $\left|f_{k}^{2}\right|_{L^{1}} \leq D_{0} \beta$. If $\lim \sup _{k \rightarrow+\infty} \mathcal{H}\left(f_{k}^{1}\right) \leq 0$, then $\mathcal{H}\left(f^{1}\right) \leq 0$ and the proof is over. If not, then

$$
\limsup _{n \rightarrow+\infty} \mathcal{H}\left(f_{k}^{2}\right) \leq-P_{1}<0
$$

We now may apply the induction hypothesis to the $L^{1}$ invariant rescaled sequence

$$
g_{k}(x, v)=f_{k}^{2}\left(\lambda_{k} x, \frac{v}{\lambda_{k}}\right) \quad \text { with } \quad \lambda_{k}=\left(\frac{1}{\left.\left.|| v\right|^{2} f_{k}^{2}\right|_{L^{1}}}\right)^{1 / 2}
$$

since this sequence satisfies

$$
\left|g_{k}\right|_{L^{1}} \leq D_{0} \beta \leq M_{1} \quad\left|g_{k}\right|_{L^{p}} \leq\left.\left. M_{p} \quad|| v\right|^{2} g_{k}\right|_{L^{1}}=1, \quad \limsup _{k \rightarrow+\infty} \mathcal{H}\left(g_{k}\right) \leq 0 .
$$

We conclude from the induction hypothesis that there exists $\hat{y}_{n}$ such that $g_{k}\left(\cdot+\hat{y}_{k}, v\right) \rightarrow$ $g$ with $|g|_{L^{1}} \geq \beta$ and $\mathcal{H}(g) \leq 0$. Observe from (5.15), $\left.\left.|| v\right|^{2} f_{k}^{2}\right|_{L^{1}} \leq 1$ and the sharp 
interpolation estimate (1.28) that

$$
1 \leq \lambda_{k} \leq P_{1}^{-1 / 2}\left(\frac{M_{1} M_{p}^{\frac{p}{p-2}}}{\left|Q_{p}\right|_{L^{1}}\left|Q_{p}\right|_{L^{p}}^{\frac{p}{p-2}}}\right)^{\frac{p-2}{4(p-1)}}<+\infty .
$$

The claim now follows from (5.16) and the construction of $f_{k}^{2}$. This concludes the proof of Lemma 5.2.

\section{Appendix}

\section{A Rescalings}

We collect here computations related to the rescalings of solutions to (1.1) in dimension $N$, that are used throughout this paper. For $1 \leq p \leq+\infty$ and $f \in \mathcal{E}_{p}$, let

$$
\tilde{f}=\gamma f\left(\frac{x}{\lambda}, \mu v\right), \quad(\gamma, \lambda, \mu) \in \mathbb{R}_{+}^{*} \times \mathbb{R}_{+}^{*} \times \mathbb{R}_{+}^{*} .
$$

We compute

$$
E_{\tilde{f}}(x)=\frac{\gamma \lambda}{\mu^{N}} E_{f}\left(\frac{x}{\lambda}\right)
$$

and

$$
\|\left.\left. v\right|^{2} \tilde{f}\right|_{L^{1}}=\left.\left.\frac{\gamma \lambda^{N}}{\mu^{N+2}}|| v\right|^{2} f\right|_{L^{1}}, \quad|\tilde{f}|_{L^{p}}=\gamma\left(\frac{\lambda}{\mu}\right)^{\frac{N}{p}}|f|_{L^{p}}, \quad\left|E_{\tilde{f}}\right|_{L^{2}}^{2}=\frac{\gamma^{2} \lambda^{N+2}}{\mu^{2 N}}\left|E_{f}\right|_{L^{2}}^{2} .
$$

\section{B Symmetric rearrangements}

In this section, some useful results on symmetric nonincreasing rearrangements are given. We refer to $[2,6,11,23]$ for details. Let us recall the definition of the Schwarz symmetrization. If $u \in L^{1}\left(\mathbb{R}^{N}\right)$ is nonnegative, we denote by $u^{*}$ the unique spherically symmetric, nonnegative, nonincreasing function such that for all $\lambda>0$

$$
\operatorname{meas}\left\{x \in \mathbb{R}^{N}: u^{*}(x) \geq \lambda\right\}=\operatorname{meas}\left\{x \in \mathbb{R}^{N}: u(x) \geq \lambda\right\} .
$$

The symmetric rearrangement has the following properties:

Lemma B.1 Let $j$ be a strictly increasing continuous function such that $j(0)=0$. Then

$$
\int_{\mathbb{R}^{N}} j\left(u^{*}\right)(x) d x=\int_{\mathbb{R}^{N}} j(u)(x) d x
$$


in particular

$$
\forall 1 \leq p \leq+\infty \quad\left|u^{*}\right|_{L^{p}}=|u|_{L^{p}}
$$

If $\psi$ is a nonnegative nondecreasing function on $\mathbb{R}_{+}$then

$$
\int_{\mathbb{R}^{N}} \psi(|x|) u^{*}(x) d x \leq \int_{\mathbb{R}^{N}} \psi(|x|) u(x) d x,
$$

and, if $\psi$ is strictly increasing, this inequality is strict unless $u^{*} \equiv u$. If $K$ is a radially symmetric strictly decreasing function then

$$
\int_{\mathbb{R}^{N}} \int_{\mathbb{R}^{N}} u(x) K(x-y) v(x) d x d y \leq \int_{\mathbb{R}^{N}} \int_{\mathbb{R}^{N}} u^{*}(x) K(x-y) v^{*}(x) d x d y,
$$

with an equality if and only if there exists $y \in \mathbb{R}^{N}$ such that $u=u^{*}(\cdot+y)$ and $v=v^{*}(\cdot+y)$.

In this paper, for distribution functions $f(x, v)$, we use either symmetric rearrangements in the $x$ variable only or in the $v$ variable only, which we respectively denote

$$
f^{* x}(\cdot, v)=(f(\cdot, v))^{*} \quad \text { and } \quad f^{* v}(x, \cdot)=(f(x, \cdot))^{*} .
$$

\section{The Cauchy problem for Vlasov-Poisson in dimension 4}

In this appendix, we consider $N=4$ and briefly sketch the proof of the fact that the lifespan of the local weak solutions built as in Theorem 1.1 is lower bounded by a function of the size of the initial data only. To wit, let $f_{0} \in \mathcal{E}_{p}$ and $f^{n}$ be a sequence of solutions to a suitable regularized problem, with initial data approximating $f_{0}$. From Diperna-Lions $[9,10]$ and the conservations of the $L^{q}$ norms and the energy, the only thing we need to prove is an a priori estimate for the kinetic energy $\left.\left.|| v\right|^{2} f^{n}\right|_{L^{1}}$ on an interval $[0, T(M)]$ (independent of $n$ ). Again, we adapt here the standard proof for the local Cauchy theory of the nonlinear Schrödinger equation in the energy space (see e.g. [6]).

Let $\left|f_{0}^{n}\right|_{\mathcal{E}_{p}} \leq M$, with $2=p_{\text {crit }}<p \leq+\infty$. We denote by $C(M)$ various constants depending only on $M$. Let

$$
\theta^{n}=\sup \left\{\tau>0:\left.\left.|| v\right|^{2} f^{n}(t)\right|_{L^{1}} \leq 2 M \text { for } 0 \leq t \leq \tau\right\} .
$$

By standard interpolation inequalities, the first moment $\rho^{n}=\int f^{n} d v$ and the second moment $j^{n}=\int v f^{n} d v$ satisfy the estimates

$$
\forall t \in\left[0, \theta^{n}\right] \quad\left|\rho^{n}(t)\right|_{L^{q}}+\left|j^{n}(t)\right|_{L^{s}} \leq C(M),
$$

with $q=\frac{6 p-4}{4 p-2}$ and $s=\frac{6 p-4}{5 p-3}$. By the equation of mass conservation $\partial_{t} \rho^{n}+\nabla_{x} \cdot j^{n}=0$, the estimate on the current $j^{n}$ yields

$$
\left|\rho^{n}\right|_{W^{1, \infty}\left(\left(0, \theta^{n}\right), W^{-1, s}\left(\mathbb{R}^{4}\right)\right)} \leq C(M) .
$$


Additionnally, by the Sobolev embedding of $L^{q}\left(\mathbb{R}^{4}\right)$ into $W^{-1, \sigma}\left(\mathbb{R}^{4}\right), \sigma=\frac{12 p-8}{5 p-2}$, we get

$$
\left|\rho^{n}\right|_{L^{\infty}\left(\left(0, \theta^{n}\right), W^{-1, \sigma}\left(\mathbb{R}^{4}\right)\right)} \leq C(M) .
$$

Let $\alpha=\frac{2 p-4}{5 p-4}$ and $\beta=\frac{12 p-8}{7 p-6}$ (remark that $p>2$ implies $\alpha \in(0,1)$ ), such that $\frac{1}{\beta}=\frac{\alpha}{s}+\frac{1-\alpha}{\sigma}$. By interpolation, we deduce from (C.2) and (C.3) that

$$
\left|\rho^{n}\right|_{C^{0, \alpha}\left(\left[0, \theta^{n}\right], W^{-1, \beta}\left(\mathbb{R}^{4}\right)\right)} \leq C(M) .
$$

Therefore, for any $t \in\left[0, \theta^{n}\right]$, we have

$$
\begin{aligned}
\left|E^{n}(t)\right|_{L^{2}}^{2}-\left|E^{n}(0)\right|_{L^{2}}^{2} & =C \int_{\mathbb{R}^{4} \times \mathbb{R}^{4}} \frac{\rho^{n}(t, x) \rho^{n}(t, y)-\rho^{n}(0, x) \rho^{n}(0, y)}{|x-y|^{2}} d x d y \\
& =C \int_{\mathbb{R}^{4} \times \mathbb{R}^{4}} \frac{\left(\rho^{n}(t, x)-\rho^{n}(0, x)\right)\left(\rho^{n}(t, y)+\rho^{n}(0, y)\right)}{|x-y|^{2}} d x d y \\
& \leq C\left|\rho^{n}(t, \cdot)-\rho^{n}(0, \cdot)\right|_{W^{-1, \beta}}\left|\frac{1}{|x|^{3}} \star\left(\rho^{n}(t, \cdot)+\rho^{n}(0, \cdot)\right)\right|_{L^{\beta^{\prime}}} \\
& \leq C t^{\alpha}\left|\rho^{n}\right|_{C^{0, \alpha}\left(\left[0, \theta^{n}\right], W^{-1, \beta}\right)}\left|\rho^{n}\right|_{L^{\infty}\left(\left(0, \theta^{n}\right), L^{q}\right)} \\
& \leq C(M) t^{\alpha},
\end{aligned}
$$

where we used (C.1), (C.4) and a Hardy-Littlewood-Sobolev inequality (indeed, $\beta^{\prime}$ was chosen such that $\left.1+\frac{1}{\beta^{\prime}}=\frac{3}{4}+\frac{1}{q}\right)$. It remains now to use the energy inequality, which yields for any $t \in\left[0, \theta^{n}\right]$

$$
\left.\left.|| v\right|^{2} f^{n}(t)\right|_{L^{1}} \leq\left.\left.|| v\right|^{2} f^{n}(0)\right|_{L^{1}}+\left|E^{n}(t)\right|_{L^{2}}^{2}-\left|E^{n}(0)\right|_{L^{2}}^{2} \leq M+C(M) t^{\alpha} .
$$

By choosing $T(M)$ such that $C(M) T(M)^{\alpha} \leq M$, we ensure that $\theta^{n} \geq T(M)$ and the proof is complete.

\section{A compactness result}

It is well-known that the Sobolev embedding $H^{1}\left(\mathbb{R}^{N}\right) \hookrightarrow L^{p}\left(\mathbb{R}^{N}\right), 2<p<2^{*}$, is not compact due to the unbounded domain, except in the special case of radial functions for $N \geq 2$ from Strauss' interpolation estimate, see [6] and references therein. For the same reason, radial solutions of the Poisson equation in dimension $N \geq 3$ depend compactly on the data; in particular, we use in this article the following result:

Lemma D.1 Let $f_{n}$ be a bounded sequence in $\mathcal{E}_{p}$ with $p \in\left(p_{\text {crit }},+\infty\right]$ such that $f_{n}$ is spherically symmetric in $x$, i.e. only depends on $|x|$ and $v$. Consider the corresponding sequence of fields given by

$$
E_{f_{n}}(x)=\frac{1}{N \omega_{N}} \int_{\mathbb{R}^{N}} \frac{x-y}{|x-y|^{N}} \rho_{f_{n}}(y) d y, \quad \rho_{f_{n}}(x)=\int_{\mathbb{R}^{N}} f_{n}(x, v) d v .
$$


Then there exists a function $f \in \mathcal{E}_{p}$ such that, up to an extraction of a subsequence, $f_{n}$ converges to $f$ in the $L^{p}\left(\mathbb{R}^{N}\right)$ weak topology (in the weak $\star$ topology in the case $p=\infty$ ) and $E_{f_{n}}$ converges to $E_{f}$ in the $L^{2}\left(\mathbb{R}^{N}\right)$ strong topology.

\section{Proof of Lemma D.1}

The first part of the result is straightforward. Let us prove that the sequence $E_{f_{n}}$ is compact in $L^{2}\left(\mathbb{R}^{N}\right)$. For $q=\frac{(N+2) p-N}{N p-N+2}$, an interpolation inequality gives

$$
\left|\rho_{f_{n}}\right|_{L^{q}} \leq\left.\left. C|| v\right|^{2} f_{n}\right|_{L^{1}} ^{\frac{N(p-1)}{2 p+N(p-1)}}\left|f_{n}\right|_{L^{p}}^{\frac{2 p}{2 p+N(p-1)}} \leq C\left|f_{n}\right|_{\mathcal{E}_{p}}
$$

which implies that $\rho_{f_{n}}$ is bounded in $L^{1} \cap L^{q}\left(\mathbb{R}^{N}\right)$. The assumption $p>p_{\text {crit }}$ ensures that $q>\frac{2 N}{N+2}$ and that for any bounded domain $\Omega, W^{1, q}(\Omega)$ is compactly embedded in $L^{2}(\Omega)$. Hence, by elliptic regularity, it is clear that the sequence $E_{f_{n}}$ is locally compact in $L^{2}\left(\mathbb{R}^{N}\right)$. To show the compactness at the infinity, it suffices to estimate the decay of $E_{f_{n}}$. The following elementary computation is adapted from [11].

If $r=|x|$, with a standard abuse of notation, we have $\rho_{f_{n}}=\rho_{f_{n}}(r), E_{f_{n}}=E_{f_{n}}(r)$ and the Poisson equation in radial coordinates gives

$$
\frac{1}{r^{N-1}} \frac{d}{r}\left(r^{N-1} E_{f_{n}}\right)=\rho_{f_{n}} .
$$

After an integration, we deduce that

$$
E_{f_{n}}(r)=\frac{1}{r^{N-1}} \int_{0}^{r} s^{N-1} \rho_{f_{n}}(r) d r \leq \frac{1}{r^{N-1}} \frac{\left|f_{n}\right|_{L^{1}}}{N \omega_{N}} .
$$

The sequence of fields $E_{f_{n}}$ is thus uniformly decreasing at the infinity and we have for any $R>0$

$$
\int_{|x|>R}\left|E_{f_{n}}\right|^{2}(x) d x \leq \frac{C}{R^{N-2}} .
$$

This completes the proof.

\section{E Proof of the concentration compactness Lemma}

This Appendix is devoted to the proof of Lemma 3.1. We follow the lines of the proof of Lemma 1.1 in [24], with adaptations to take into account the compactness information in $v$ from the uniform control of the kinetic energy (3.1).

Let the concentration function

$$
\mathcal{C}_{n}(R)=\sup _{Y \in \mathbb{R}^{2 N}} \int_{Y+B_{R}} f_{n}(X) d X
$$


then $\left(\mathcal{C}_{n}\right)_{n \geq 1}$ is a sequence of non decreasing, nonnegative, uniformly bounded functions on $\mathbb{R}^{+}$with $\lim _{R \rightarrow+\infty} \mathcal{C}_{n}(R)=M$. Thus from standard argument, there exists a subsequence $\left(f_{n_{k}}\right)_{k \geq 1}$ and a nondecreasing nonnegative function $\mathcal{C}$ such that $\mathcal{C}_{n_{k}}(R) \rightarrow \mathcal{C}(R)$ for all $R \geq 0$ as $n \rightarrow+\infty$. Moreover,

$$
m=\lim _{R \rightarrow+\infty} \mathcal{C}(R) \quad \text { belongs to } \quad[0, M]
$$

Step 1 Vanishing.

Assume $m=0$, then vanishing occurs. Indeed, we have $\mathcal{C} \equiv 0$, thus

$$
\forall R>0, \quad \lim _{k \rightarrow+\infty} \sup _{Y \in \mathbb{R}^{2 N}} \int_{Y+B_{R}} f_{n_{k}}(X) d X=0 .
$$

Now pick $\varepsilon>0$. From (3.1), there exists $R(\varepsilon) \in(0,+\infty)$ such that

$$
\forall n \geq 1, \quad \int_{x \in \mathbb{R}^{N}} \int_{|v| \geq R(\varepsilon)} f_{n}(x, v) d v d x \leq \frac{\sup _{n} \iint|v|^{2} f_{n}(x, v) d v d x}{R^{2}(\varepsilon)}<\varepsilon .
$$

Let $\tilde{R}>0$, then

$$
\begin{aligned}
\int_{y+B_{\tilde{R}}} \rho_{n_{k}}(x) d x & =\int_{y+B_{\tilde{R}}} \int_{|v|<R(\varepsilon)} f_{n_{k}}(x, v) d v d x+\int_{y+B_{\tilde{R}}} \int_{|v| \geq R(\varepsilon)} f_{n_{k}}(x, v) d v d x \\
& \leq \sup _{Y \in \mathbb{R}^{2 N}} \int_{Y+B_{\tilde{R}+R(\varepsilon)}} f_{n_{k}}(X) d X+\varepsilon
\end{aligned}
$$

Taking the sup in $y \in \mathbb{R}^{N}$ and letting $k \rightarrow+\infty$, we conclude from (E.2)

$$
\forall \tilde{R}>0, \quad \lim _{k \rightarrow+\infty} \sup _{y \in \mathbb{R}^{N}} \int_{y+B_{\tilde{R}}} \rho_{n_{k}}(x) d x \leq \varepsilon,
$$

and as this holds for any $\varepsilon>0,(3.3)$ follows.

Step 2 Compactness.

Assume now $m=M$, then compactness occurs. Indeed, let $\frac{M}{2} \leq \mu<M$, then there exists $R(\mu) \in(0,+\infty)$ such that

$$
\forall k \geq 1, \quad \mathcal{C}_{n_{k}}(R(\mu))=\sup _{Y \in \mathbb{R}^{2 N}} \int_{Y+B_{R(\mu)}} f_{n_{k}}(X) d X>\mu,
$$

and thus there exists $Y_{k}(\mu) \in \mathbb{R}^{2 N}$ such that

$$
\int_{Y_{k}(\mu)+B_{R(\mu)}} f_{n_{k}}(X) d X>\mu
$$


We then have

$$
\int_{Y_{k}(\mu)+B_{R(\mu)}} f_{n_{k}}(X) d X+\int_{Y_{k}\left(\frac{M}{2}\right)+B_{R\left(\frac{M}{2}\right)}} f_{n_{k}}(X) d X>\mu+\frac{M}{2}>M
$$

and thus $B\left(Y_{k}\left(\frac{M}{2}\right), R\left(\frac{M}{2}\right)\right) \cap B\left(Y_{k}(\mu), R(\mu)\right) \neq \emptyset$ from which

$$
\forall \mu \in\left[\frac{M}{2}, M\right), \quad\left|Y_{k}\left(\frac{M}{2}\right)-Y_{k}(\mu)\right| \leq R\left(\frac{M}{2}\right)+R(\mu) .
$$

Letting $\tilde{R}(\mu)=R\left(\frac{M}{2}\right)+2 R(\mu)$, we conclude

$$
\forall \mu \in\left[\frac{M}{2}, M\right), \quad \int_{Y_{k}\left(\frac{M}{2}\right)+B_{\tilde{R}(\mu)}} f_{n_{k}}(X) d X>\mu .
$$

Letting $Y_{k}=\left(y_{k}, v_{k}\right)$,

$$
\int_{y_{k}\left(\frac{M}{2}\right)+B_{\tilde{R}(\mu)}} \rho_{n_{k}}(x) d x \geq \int_{Y_{k}\left(\frac{M}{2}\right)+B_{\tilde{R}(\mu)}} f_{n_{k}}(X) d X>\mu
$$

and (3.2) follows.

Step 3 Dichotomy.

Assume now $m \in(0, M)$, then dichotomy occurs. Pick $0<\varepsilon \ll m$ and let $R(\varepsilon)$ such that (E.3) holds together with

$$
\mathcal{C}(R(\varepsilon))>m-\varepsilon
$$

We have

$$
m-\varepsilon<\mathcal{C}_{n_{k}}(R(\varepsilon))<m+\varepsilon \text { for } k \geq k_{0}
$$

large enough, and thus there exists $Y_{k} \in \mathbb{R}^{2 N}$ such that

$$
m-\varepsilon<\int_{Y_{k}+B_{R(\varepsilon)}} f_{n_{k}}(X) d X<m+\varepsilon .
$$

Moreover, there exists a sequence $\left(R_{k}\right)_{k \geq k_{0}}$ with

$$
\mathcal{C}_{n_{k}}\left(R_{k}\right) \leq m+\varepsilon \text { and } R_{k} \rightarrow+\infty \text { as } k \rightarrow+\infty
$$

and thus from (E.4)

$$
\begin{aligned}
\int_{R(\varepsilon) \leq\left|Y-Y_{k}\right| \leq R_{k}} f_{n_{k}}(X) d X & =\int_{Y_{k}+B_{R_{k}}} f_{n_{k}}(X) d X-\int_{Y_{k}+B_{R(\varepsilon)}} f_{n_{k}}(X) d X \\
& \leq(m+\varepsilon)-(m-\varepsilon) \leq 2 \varepsilon .
\end{aligned}
$$


Let $Y_{k}=\left(y_{k}, v_{k}\right)$, we have from (E.3) and (E.4)

$$
\left|v_{k}\right| \leq 2 R(\varepsilon) .
$$

Taking $k_{0}$ such that $R_{k}>3 R(\varepsilon)$ for any $k \geq k_{0}$, consider now

$$
f_{k}^{1}(x, v)=f_{n_{k}}(x, v) \mathbf{1}_{\left|x-y_{k}\right|<3 R(\varepsilon)}, \quad f_{k}^{2}(x, v)=f_{n_{k}}(x, v) \mathbf{1}_{\left|x-y_{k}\right|>R_{k}}
$$

so that

$$
\rho_{k}^{1}(x)=\rho_{n_{k}}(x) \mathbf{1}_{\left|x-y_{k}\right|<3 R(\varepsilon)}, \quad \rho_{k}^{2}(x)=\rho_{n_{k}}(x) \mathbf{1}_{\left|x-y_{k}\right|>R_{k}},
$$

then from (E.6),

$$
\begin{aligned}
& \left|\int_{y_{k}+B_{3 R(\varepsilon)}} \rho_{n_{k}}(x) d x-\int_{Y_{k}+B_{3 R(\varepsilon)}} f_{n_{k}}(X) d X\right|+\left|\int_{y_{k}+B_{R_{k}}} \rho_{n_{k}}(x) d x-\int_{Y_{k}+B_{R_{k}}} f_{n_{k}}(X) d X\right| \\
& \quad \leq 2 \iint_{|v| \geq R(\varepsilon)} f_{n_{k}}(x, v) d v d x<2 \varepsilon
\end{aligned}
$$

(the norm chosen on $\mathbb{R}^{2 N}$ is $|(x, v)|=\max (|x|,|v|)$ ). From this inequality it can be deduced two facts: first, with (E.5),

$$
\left|\rho_{n_{k}}-\rho_{k}^{1}-\rho_{k}^{2}\right|_{L^{1}}=\int_{y_{k}+B_{R_{k}}} \rho_{n_{k}}(x) d x-\int_{y_{k}+B_{3 R(\varepsilon)}} \rho_{n_{k}}(x) d x \leq 4 \varepsilon ;
$$

second, with (E.4) and (E.5),

$$
\left|\int_{\mathbb{R}^{N}} \rho_{k}^{1}(x) d x-m\right| \leq\left|\int_{Y_{k}+B_{3 R(\varepsilon)}} f_{n_{k}}(x) d x-m\right|+2 \varepsilon \leq 5 \varepsilon .
$$

Hence (3.4) follows, which concludes the proof of Lemma 3.1.

Acknowledgement. We acknowledge support by the European IHP network no. RNT2 2001349 entitled "Hyperbolic and kinetic equation : asymptotics, numerics, applications", by the ACI Nouvelles Interfaces des Mathématiques no. ACINIM 176-2004 funded by the French ministry of research as well as the ACI Jeunes chercheurs no. JC1035.

\section{References}

[1] Batt, J.; Faltenbacher, W.; Horst, E., Stationary spherically symmetric models in stellar dynamics, Arch. Rat. Mech. Anal. 93 (1986), 159-183.

[2] Berestycki, H.; Lions, P.-L., Nonlinear scalar field equations. I. Existence of a ground state, Arch. Rational Mech. Anal. 82 (1983), no. 4, 313-345.

[3] Binney, J.; Tremaine, S., Galactic Dynamics, Princeton University Press, 1987. 
[4] Bobylev, A. V.; Dukes, P.; Illner, R.; Victory, H. D., On Vlasov-Manev Equations. I. Foundations, Properties, and Nonglobal Existence, J. Stat. Phys. 88 (1997), no. 3-4, $885,911$.

[5] Bouchut, F.; Golse, F.; Pulvirenti, M., Kinetic equations and asymptotic theory, Series in Appl. Math., Gauthiers-Villars, Paris, 2000.

[6] Cazenave, T., Semilinear Schrödinger equations, Courant Lecture Notes in Mathematics, 10, NYU, CIMS, AMS 2003.

[7] Cazenave, T.; Lions, P.-L., Orbital stabiity of standing waves for some nonlinear Schrödinger equations, Comm. Math. Phys. 85 (1982), no. 4, 549-561.

[8] Chavanis, P.-H., Statistical mechanics and thermodynamic limit of self-gravitating fermions in $D$ dimensions, Physical Review E 69 (2004), 066126.

[9] Diperna, R. J.; Lions, P.-L., Global weak solutions of kinetic equations, Rend. Sem. Mat. Univ. Politec. Torino 46 (1988), no. 3, 259-288 (1990).

[10] Diperna, R. J.; Lions, P.-L., Solutions globales d'équations du type Vlasov-Poisson, C. R. Acad. Sci. Paris Sér. I Math. 307 (1988), no. 12, 655-658.

[11] Dolbeault, J.; Sánchez, Ó.; Soler, J., Asymptotic behaviour for the Vlasov-Poisson system in the stellar-dynamics case, Arch. Ration. Mech. Anal. 171 (2004), no. 3, $301-327$

[12] Fridmann, A. M.; Polyachenko, V. L., Physics of gravitating systems, Springer-Verlag, New York, 1984.

[13] Gidas, B.; Ni, W.M.; Nirenberg, L., Symmetry and related properties via the maximum principle, Comm. Math. Phys. 68 (1979), 209-243.

[14] Ginibre, J.; Velo, G., On a class of nonlinear Schrödinger equations. I. The Cauchy problem, general case. J. Funct. Anal. 32 (1979), no. 1, 1-32.

[15] Glassey, R., The Cauchy problem in kinetic theory, Society for Industrial and Applied Mathematics (SIAM), Philadelphia, PA, 1996.

[16] Guo, Y., Variational method for stable polytropic galaxies, Arch. Rat. Mech. Anal. 130 (1995), 163-182.

[17] Guo, Y.; Rein, G., Stable steady states in stellar dynamcics, Arch. Rat. Mech. Anal. 147 (1999), 225-243.

[18] Guo, Y.; Rein, G., Existence and stability of Camm type steady states in galactic dynamics, Indiana Univ. Math. J. 48 (1999), 1237-1255. 
[19] Guo, Y.; Rein, G., Isotropic steady states in galactic dynamics, Comm. Math. Phys. 219 (2001), 607-629.

[20] Horst, E.; Hunze, R., Weak solutions of the initial value problem for the unmodified nonlinear Vlasov equation, Math. Methods Appl. Sci. 6 (1984), no. 2, 262-279.

[21] Kwong, M. K., Uniqueness of positive solutions of $\Delta u-u+u^{p}=0$ in $R^{n}$. Arch. Rational Mech. Anal. 105 (1989), no. 3, 243-266.

[22] Lemou, M.; Méhats, F.; Raphael, P., Orbital stability and singularity formation for Vlasov Poisson systems, C. R. Math. Acad. Sci. Paris 341 (2005), no. 4, 269-274.

[23] Lieb, E.; Loss, M., Analysis, Graduate Studies in Mathematics, 14, American Mathematical Society, Providence, RI, 1997.

[24] Lions, P.-L., The concentration-compactness principle in the calculus of variations. The locally compact case. I. Ann. Inst. H. Poincaré Anal. Non Linéaire 1 (1984), no. $2,109-145$.

[25] Lions, P.-L., The concentration-compactness principle in the calculus of variations. The locally compact case. II. Ann. Inst. H. Poincaré Anal. Non Linéaire 1 (1984), no. $4,223-283$.

[26] Lions, P.-L.; Perthame, B., Propagation of moments and regularity for the 3dimensional Vlasov-Poisson system. Invent. Math. 105 (1991), no. 2.

[27] Martel, Y.; Merle, F., Asymptotic stability of solitons for subcritical generalized KdV equations. Arch. Ration. Mech. Anal. 157 (2001), no. 3, 219-254.

[28] Martel, Y.; Merle, F.; Tsai, T.P., Stability and asymptotic stability in the energy space of the sum of $N$ solitons for subcritical gKdV equations, Comm. Math. Phys. 231 (2002), no. 2, 347-373.

[29] Merle, F., Lower bounds for the blow up rate of solutions of the Zakharov equations in dimension two, Comm. Pure. Appl. Math. 49 (1996), n0. 8, 765-794.

[30] Merle, F.; Raphaël, P., Blow up dynamic and upper bound on the blow up rate for critical nonlinear Schrödinger equation, Annals of Math. 161 (2005), no. 1, 157-222.

[31] Merle, F.; Raphaël, P., On Universality of Blow up Profile for $L^{2}$ critical nonlinear Schrödinger equation, Invent. Math. 156 (2004), 565-672.

[32] Merle, F.; Raphaël, P., Profiles and quantization of the blow up mass for critical non linear Schrödinger equation, Comm. Math. Phys. 253 (2004), n.3, 675-704.

[33] Merle, F.; Tsutsumi, Y., $L^{2}$ concentration of blow up solutions for the nonlinear Schrödinger equation with critical power nonlinearity, J.Diff.Eq. 84 (1990), 205-214. 
[34] Nawa, H., "Mass concentration" phenomenon for the nonlinear Schrödinger equation with the critical power nonlinearity, Funkcial. Ekvac. 35 (1992), no. 1, 1-18.

[35] Rein, G., Flat steady states in stellar dynamics - Existence and stability, Comm. Math. Phys. 205 (1999), 229-247.

[36] Sánchez, Ó.; Soler, J., Orbital stability for polytropic galaxies, preprint.

[37] Schaeffer, J., Steady States in Galactic Dynamics, Arch. Rational, Mech. Anal. 172 (2004), 1-19.

[38] Weinstein, M.I., Nonlinear Schrödinger equations and sharp interpolation estimates, Comm. Math. Phys. 87 (1983), 567-576.

[39] Zakharov, V.E.; Shabat, A.B., Exact theory of two-dimensional self-focusing and onedimensional self-modulation of waves in non-linear media, Sov. Phys. JETP 34 (1972), $62-69$. 